\title{
Quantifying sustainability indicators in striped catfish Pangasianodon hypophthalmus downstream and upstream ponds
}

\author{
N. Nhut ${ }^{1,2}$, N. V. Hao ${ }^{1}$, R. H. Bosma ${ }^{2}$, J. A. V. Verreth ${ }^{2}$, M. C. J. Verdegem ${ }^{2, *}$, \\ E. H. Eding ${ }^{2}$
}

\begin{abstract}
${ }^{1}$ Research Institute for Aquaculture No. 2, 116 Nguyen Dinh Chieu, District 1, HCM City, Vietnam
${ }^{2}$ Aquaculture and Fisheries Group, Wageningen University, 6700AH Wageningen, The Netherlands
\end{abstract}

\begin{abstract}
To assess the potential for improving sustainability and efficiency in an important Vietnamese finfish culture, we quantified sustainability indicators for 2 downstream and 2 upstream 3 to $4 \mathrm{~m}$ deep ponds for striped catfish Pangasianodon hypophthalmus (Sauvage, 1878) production along the Mekong River, Vietnam. The resources used per kg fish produced were (downstream vs. upstream): 2.8 vs. $7.1 \mathrm{~m}^{3}$ water, $0.04 \mathrm{vs.} 0.14 \mathrm{kWh}$ energy, $0.06 \mathrm{vs} .0 .11 \mathrm{~h}$ human labour and 0.06 vs. $0.15 \mathrm{~g}$ antibiotics $(\mathrm{p}<0.05)$. The feed conversion ratio (kg feed dry matter per $\mathrm{kg}$ fish wet weight) was 1.50 vs. 1.65 and feed dry matter utilization efficiency was similar (28 vs. $30 \%$ ) for both types of ponds ( $p>0.05$ ). Nitrogen and phosphorus utilization efficiencies were 44 vs. $40 \%$ for nitrogen ( $p<0.05$ ) and 17.7 vs. $17.6 \%$ for phosphorus $(p>0.05)$. An advantage of production in deep ponds is the breakdown of organic matter through denitrification and fermentation. Based on the difference between feed input and monitored outputs and sinks, an estimated $29-37 \%$ of dry matter volatilized as $\mathrm{CO}_{2}$ and $30-34 \%$ of nitrogen was lost as $\mathrm{N}_{2}$. A major disadvantage of the deep pond production system is its dependency on water exchange with the Mekong River, resulting in low biosecurity, which is aggravated by the lack of availability of disease-free fingerlings. Dealing with disease caused farmers to apply suboptimal feeding rations and to lengthen the culture period. Developing (semi-)closed systems that adopt elements from recirculation technology could improve both culture performance and sustainability.
\end{abstract}

KEY WORDS: Pangasius - Striped catfish - Nutrient budget - Water quality - Waste effluent · Mass balance

\section{INTRODUCTION}

Striped catfish Pangasianodon hypophthalmus (Sauvage, 1878) farming in the Mekong Delta of Vietnam has developed rapidly into one of the country's major aquaculture industries (Phuong \& Oanh 2010). In 2016, total catfish production reached 1.1 million tonnes produced in 5500 ha of ponds. About 600000 tonnes of processed pangasius products have been exported to more than 150 countries (MARD 2016).

With a production of 70-800 metric tonnes (MT) $\mathrm{ha}^{-1} \mathrm{yr}^{-1}$ (Phan et al. 2009), striped catfish culture is

\footnotetext{
${ }^{*}$ Corresponding author: marc.verdegem@wur.nl
}

intensive. Water quality is maintained through water exchange. Water use is an important sustainability indicator. The Aquaculture Stewardship Council (ASC) has set the not-to-exceed standard at $5000 \mathrm{l}$ $\mathrm{kg}^{-1}$ striped catfish (ASC 2012). Water use values reported in the literature are either $20-50 \%$ smaller (2500-4050 $\mathrm{l} \mathrm{kg}^{-1}$ fish; Bosma et al. 2009, Phan et al. 2009) or $82 \%$ larger (9167 l kg-1 fish; Anh et al. 2010). ASC indicators for nitrogen $(\mathrm{N})$ and phosphorus (P) discharge are $27.5 \mathrm{~g} \mathrm{~N}$ and $7.2 \mathrm{~g} \mathrm{P} \mathrm{kg}^{-1}$ fish (ASC 2012). These ASC-indicator values are substantially lower than the estimated values for $\mathrm{N}$ and $\mathrm{P}$ dis-

(C) The authors 2017. Open Access under Creative Commons by Attribution Licence. Use, distribution and reproduction are unrestricted. Authors and original publication must be credited. 
charge reported in the literature: $38-46 \mathrm{~g} \mathrm{~N} \mathrm{~kg}^{-1}$ (Anh et al. 2010, De Silva et al. 2010) and 9.9-14.4 g $\mathrm{P} \mathrm{kg}^{-1}$ fish produced (De Silva et al. 2010).

Today, the common practice is to culture striped catfish in 2-6 $\mathrm{m}$ deep earthen ponds close to the Mekong River (Phan et al. 2009). Striped catfish farming started upstream (US) before expanding to downstream (DS) areas. Both US and DS farmers practice tidal water exchange. DS farmers have the advantage of a larger tidal amplitude and less potential conflict with other types of land use (Bosma et al. 2009). In addition, DS farmers use less energy than US farmers, because in contrast to the latter, they do not have to pump to realize sufficient water exchange. However, higher water exchange might also result in more waste discharge. Thus, when analyzing the sustainability of current striped catfish farming technology, it is important to quantify the nutrient discharge related to water exchange. The objective of this study was to quantify the water exchange, nutrient utilization efficiency and sustainability indicators in striped catfish US and DS ponds in the Mekong Delta. To improve the sustainability of striped catfish production it is important to get insights into water and nutrient utilization over a complete culture period (ASC 2012). Many studies have already reported nutrient utilization, water use and energy use of striped catfish ponds during a full production cycle (e.g. Bosma et al. 2009, De Silva et al. 2010), but have not measured parameters at the level of detail presented in the current study. In addition, differences between DS and US ponds have not been previously reported. A comparison was made to reported nutrient and water budgets for other major aquaculture species and options to improve sustainability of striped catfish pond farming are suggested.

\section{MATERIALS AND METHODS}

\section{Experimental ponds}

Four ponds were used, 2 per farm: one farm located DS and the other located US in the Mekong Delta, Vietnam. The 2 DS ponds were located at the Phuoc Binh hamlet, Quoi Thien commune, Vung Liem district, Vĩnh Long province, $63 \mathrm{~km}$ apart from the 2 US ponds at the My Suong hamlet, Cao Lanh district, Dong Thap province. The ponds were newly constructed and had a surface area of $1.11 \pm 0.1$ ha at the DS and $1.08 \pm 0.1$ ha at the US farm (Table 1). Because of the high sampling frequency and the fact that the large number of parameters measured re- quired extensive manpower and a permanent presence at each of the study ponds, there was insufficient capacity and budget to sample more than 4 ponds. The selected ponds were representative of farming practices in DS and US locations in this part of the Mekong Delta.

\section{Pond management}

The 2 DS and 2 US ponds were operated following the farm's protocol and monitored during a full production cycle. Fish were stocked at a water depth between 2.5 and $3.0 \mathrm{~m}$; the water was raised to a depth of 3.4-4.2 $\mathrm{m} 1 \mathrm{mo}$ after stocking. This depth was maintained until harvest. In DS ponds, tidal water exchange was practiced, whereas in US ponds a combination of tidal exchange and active pumping was used. No aeration was applied during culture. Pond management included control of water exchange, liming and sludge removal (Table 1, Fig. 1).

\section{Fish, feed and feeding}

Striped catfish Pangasianodon hypophthalmus fingerlings were stocked at a density of 51.0 ind. $\mathrm{m}^{-2}$ in DS ponds and at 51.3 ind. $\mathrm{m}^{-2}$ in US ponds, with a mean individual weight of 46 and $31 \mathrm{~g}$, respectively. The same commercial extruded feeds were applied in all ponds (Viet Thang Company). During each of 3 distinct periods in the culture cycle (Days 1-59, 60-122, 123 to harvest), a diet with different composition and pellet size was fed (Table 2). The fish were hand-fed daily at 10:00 $\mathrm{h}$. The farmers aimed to feed $2-3 \%$ body weight $\mathrm{d}^{-1}$. For each distinct period, feed samples from 5 randomly selected $25 \mathrm{~kg}$ feed bags were pooled to determine the proximate composition (Table 2).

\section{Sampling and measurement}

\section{Sampling}

During the production cycle, pond influent (river water) and pond water were sampled biweekly (see 'Measurements and analyses' below). Water in each pond was sampled at 5 locations, after which samples were mixed into one composite sample following the procedure described by Nhan et al. (2008). The influent water was sampled next to the inlet sluice gate in the river. Water samples were collected in a PVC 
Table 1. Pond characteristics and management in downstream (DS) and upstream (US) striped catfish ponds. Labour use included farm activities of feeding, water exchange, fish health monitoring, water quality monitoring, sludge removal, fish transport, feed transport, log keeping, and accounting; the labour for transport, log keeping and accounting were classified as indirect labour. Lime was applied to maintain $\mathrm{pH}$ and alkalinity during culture; antibiotics were applied during fish disease outbreaks but not during the 2 mo before harvest (Fig. 2). Electricity from the public grid was used. Mean \pm SD $(n=2)$, where applicable

\begin{tabular}{|c|c|c|}
\hline Parameter & Downstream & Upstream \\
\hline Pond depth (m) & $3.4 \pm 0.1$ & $4.2 \pm 0.1$ \\
\hline Pond surface area (ha) & $1.1 \pm 0.1$ & $1.1 \pm 0.1$ \\
\hline Pumping & $\begin{array}{l}\left.2 \text { diesel pumps (8 HP pump }{ }^{-1}\right) ; \\
\text { no electric pump }\end{array}$ & $\begin{array}{l}1 \text { electric pumping station ( } 30 \mathrm{HP}), 5-8 \mathrm{~h} \mathrm{~d}^{-1} \\
\text { at neap tide and } 1-2 \mathrm{~h} \mathrm{~d}^{-1} \text { at spring tide }\end{array}$ \\
\hline \multicolumn{3}{|l|}{ Water exchange } \\
\hline Timing & $\begin{array}{c}\text { Discharge at low tide, intake at high tide, } \\
\text { no exchange during neap tide }\end{array}$ & $\begin{array}{l}\text { Discharge at low tide, intake at high tide, } \\
\text { exchange through pumping during neap tide }\end{array}$ \\
\hline Daily exchange (\%) & In first 3 mo: $0-10$ & In first 2 mo: $0-10$ \\
\hline & At harvest: $10-40$ & At harvest: $12-50$ \\
\hline Time to refill (h) & $8-12$ & $6-8$ \\
\hline \multicolumn{3}{|l|}{ Sludge removal } \\
\hline Volume $\left(\mathrm{m}^{3}\right)$ & $3199 \pm 842$ & $3200 \pm 212$ \\
\hline Percent dry matter & $2.1 \pm 0.4$ & $2.6 \pm 0.6$ \\
\hline Frequency & 3 times & Once \\
\hline Labour use pond ${ }^{-1}$ & 3 farm employees, 1 technician & 3 farm employees, 1 technician \\
\hline \multicolumn{3}{|l|}{ Chemical use } \\
\hline Lime $\left(\mathrm{kg} \mathrm{CaCO}_{3} \mathrm{ha}^{-1}\right)$ & 1000 & 2100 \\
\hline Iodine $(10 \%)$ & $0.5-1$ & $0.5-1$ \\
\hline $\mathrm{NaCl}$ (table salt; ppm) & $10-15$ & $10-15$ \\
\hline \multicolumn{3}{|l|}{ Antibiotic use ( $\mathrm{g} \mathrm{kg}^{-1}$ feed) } \\
\hline Doxycycline & $2.5-5$ & $2.5-5$ \\
\hline Oxytetracycline & $2.5-5$ & $2.5-5$ \\
\hline Florphenicol & $2-6$ & $2-6$ \\
\hline \multicolumn{3}{|l|}{ Energy/fuel use } \\
\hline Electricity & $\begin{array}{c}\text { Light during nighttime, on farm lodges, office } \\
\text { and operation }\end{array}$ & $\begin{array}{l}\text { Water exchange; light during nighttime, on } \\
\text { farm lodges, office and operation }\end{array}$ \\
\hline Diesel & Sludge removal, (emergency) pumping & Sludge removal, emergency pumping \\
\hline
\end{tabular}

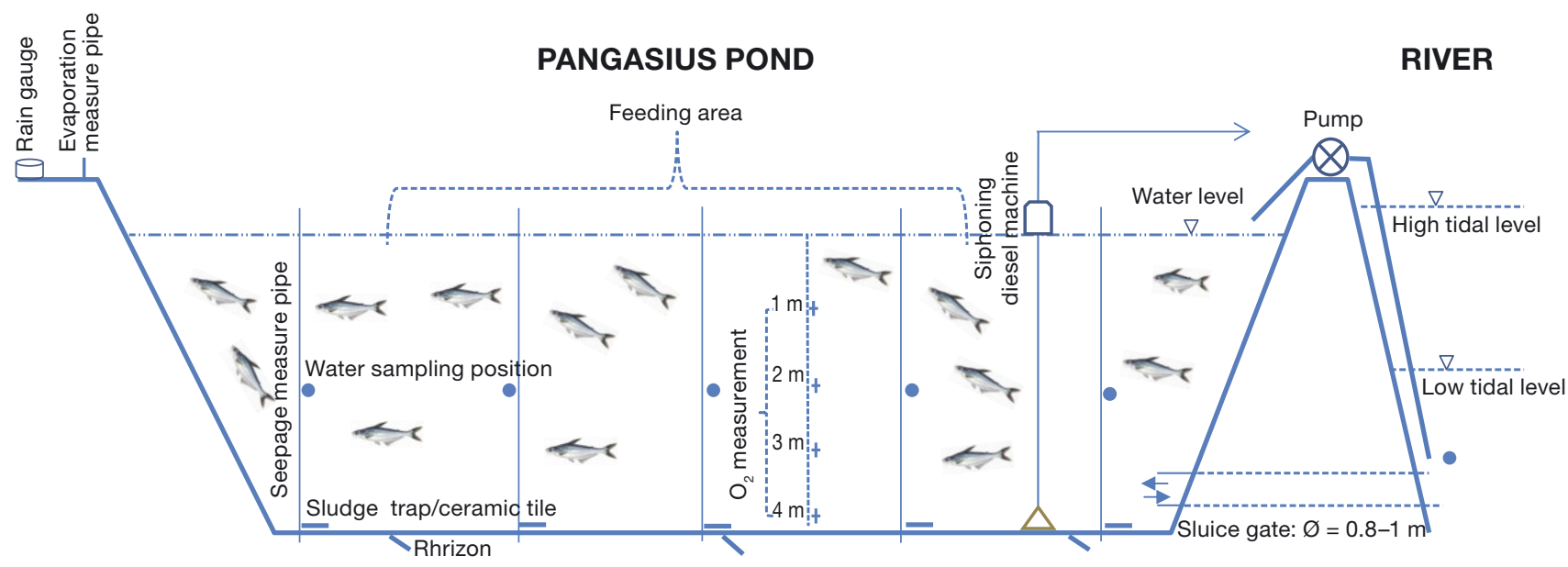

Fig. 1. Schematic view of experimental striped catfish ponds. Number of sample locations for sludge (fixed) and water column samples (random) are shown. Oxygen measurements were taken at fixed depths at random locations. Evaporation and rain volume measurements were taken on the dike. Position of the sluice gate was $0.5 \mathrm{~m}$ above the bottom (inner sluice valve not shown). Pumps for water exchange were positioned on top of the dam next to the river, and located in the corner away from the sluice gate. When sludge was siphoned from the bottom, 3 floating movable diesel pumps were used (see also Table 1) 
Table 2. Diet composition, pellet size and amount of feed used in 3 different culture periods of the pangasius production cycle. Nutrient content was analyzed on wet weight basis ( $\mathrm{g} \mathrm{kg}{ }^{-1}$ feed). COD: chemical oxygen demand; NFE: nitrogen-free extract; DS: downstream; US: upstream. COD content of the diet was calculated as described in Dalsgaard \& Pedersen (2011): COD feed $=$ crude protein $\left(\mathrm{g} \mathrm{kg}^{-1}\right) \times 1.77+$ crude fat $\left(\mathrm{g} \mathrm{kg}^{-1}\right) \times 2.88+\mathrm{NFE}\left(\mathrm{g} \mathrm{kg}^{-1}\right) \times 1.16+$ fiber $\left(\mathrm{g} \mathrm{kg}^{-1}\right) \times 1.16$. Energy content of the diet was calculated as $3.4 \mathrm{kcal} \mathrm{g}^{-1}$ COD according to Henken et al. (1986)

\begin{tabular}{|c|c|c|c|c|c|c|}
\hline \multirow[t]{2}{*}{ Parameter } & \multicolumn{2}{|c|}{$\begin{array}{c}\text { Period } 1 \\
\text { (Day 1-59) }\end{array}$} & \multicolumn{2}{|c|}{$\begin{array}{c}\text { Period } 2 \\
\text { (Day 60-122) }\end{array}$} & \multicolumn{2}{|c|}{$\begin{array}{c}\text { Period 3 } \\
\text { (Day 122-harvest) }\end{array}$} \\
\hline & DS & US & DS & US & DS & US \\
\hline Pellet diameter (mm) & 2.5 & 2.5 & 4.0 & 4.0 & 8.0 & 8.0 \\
\hline Total feed $\left(10^{3} \mathrm{~kg}\right)$ & 17.95 & 8.55 & 79.18 & 26.83 & 389.25 & 353.54 \\
\hline Dry matter ( $\mathrm{g} \mathrm{kg}^{-1}$ feed) & 889.1 & 891.9 & 894.0 & 894.0 & 892.0 & 894.0 \\
\hline Crude protein ( $\mathrm{g} \mathrm{kg}^{-1}$ feed) & 303.6 & 300.7 & 282.0 & 280.0 & 262.0 & 263.0 \\
\hline Crude fat ( $\mathrm{g} \mathrm{kg}^{-1}$ feed) & 52.0 & 52.0 & 53.0 & 53.0 & 52.0 & 54.0 \\
\hline NFE $\left(g_{k g}^{-1}\right.$ feed $)$ & 406.3 & 405.9 & 419.0 & 416.0 & 431.0 & 424.0 \\
\hline Fiber ( $\mathrm{g} \mathrm{kg}^{-1}$ feed) & 54.2 & 59.2 & 67.0 & 68.0 & 73.0 & 76.0 \\
\hline Ash $\left(\mathrm{g} \mathrm{kg}^{-1}\right.$ feed $)$ & 73.0 & 74.1 & 73.0 & 77.0 & 74.0 & 77.0 \\
\hline Total-P ( $\mathrm{g} \mathrm{kg}^{-1}$ feed) & 13.3 & 13.2 & 13.2 & 13.5 & 14.0 & 13.4 \\
\hline COD (g kg ${ }^{-1}$ feed $)$ & 1221.4 & $1,216.9$ & 1215.5 & 1209.7 & 1198.1 & 1201.0 \\
\hline Energy (kcal kg-1 feed) & 4157.7 & 4137.5 & 4132.8 & 4112.9 & 4073.7 & 4083.5 \\
\hline
\end{tabular}

pipe (5.8 $\mathrm{cm}$ inner diameter) with the length adjusted to the pond depth; the pipe was lowered vertically at each sampling site, covering the full water column. Once the pipe was in position, the bottom opening was closed by a stopper pulled in place by a rope passing through the pipe. In the river, a $3 \mathrm{~m}$ long pipe with the same diameter was used to sample the water column between 1.5 and 4.5 m depth (Fig. 1). Rhizon pore water samplers (Eijkelkamp Agrisearch Equipment) were installed $0.3 \mathrm{~m}$ deep in the sediment at 3 randomly chosen locations in each pond to collect seepage water as described by Muendo et al. (2005). The collected rhizon water samples were mixed into one composite sample. Precipitation was measured daily at DS and US locations.

Every fortnight, 50 fish pond ${ }^{-1}$ were caught by casting net, and batch weighed to determine the average individual weight. Of these 50 fish, 15 were randomly taken to determine proximate composition; the remaining fish were returned to the pond. At harvest, all fish were removed by a processor, of which 15 fish pond ${ }^{-1}$ were processed to record fillet yield and fillet coloration, and another 15 fish were analyzed for proximate composition.

At 3 randomly selected locations in each pond, a $0.6 \mathrm{~m}^{2}$ circular sludge trap and a $0.4 \mathrm{~m}^{2}$ ceramic tile were installed horizontally on the sediment surface. Sludge traps were emptied on a weekly basis, because they would spill over when sampled biweekly. However, the amount of sludge collected was reported on a biweekly basis. The areas with tiles were marked and sediment accumulating above each tile was left undisturbed until harvest. On the day of harvest, the accumulated sludge above the tiles was quantified and analyzed. Farmers decided when to remove sludge that accumulated at the bottom in the pond (Table 1). Sludge removal was done using a diesel powered suction pump when the sludge bed grew higher than $20 \mathrm{~cm}$ (checked by divers). When this happened, a sludge sample was collected and the total volume removed was recorded. Thus, the total amount and composition of the sludge removed could be determined.

\section{Measurements and analyses}

Water. Dissolved oxygen and $\mathrm{pH}$ were measured daily at $06: 00 \mathrm{~h}$ at $3.5 \mathrm{~m}$ depth in the river next to the inlet of the sluice gate (Fig. 1). Inside each pond, water temperature $\left({ }^{\circ} \mathrm{C}\right)$, dissolved oxygen $\left(\mathrm{mg} \mathrm{l}^{-1}\right)$ and $\mathrm{pH}$ were measured by a multi-parameter meter (HI9828, Hanna Instruments). Secchi disk transparency $(\mathrm{cm})$ (Almazan \& Boyd 1978) was measured daily at 06:00 and 14:00 $\mathrm{h}$ at 5 randomly chosen locations at 1, 2, 3 and $4 \mathrm{~m}$ depth (Fig. 1).

The collected water samples in river and ponds, including rain and seepage water, were analyzed according to APHA (1999) for chemical oxygen demand (COD; dichromate reflux, $0.45 \mu \mathrm{m}$ filter pore size), $5 \mathrm{~d}$ biological oxygen demand $\left(\mathrm{BOD}_{5}\right)$, total organic carbon (TOC; high temperature combustion method), total carbon ( $\mathrm{TC}_{i}$ high temperature combustion method), carbon dioxide $\left(\mathrm{CO}_{2}\right.$ f free $\mathrm{CO}_{2}$ reacts with sodium hydroxide to form sodium bicarbonate), total alkalinity (titration with sulfuric acid and methyl orange indicator), total Kjeldahl nitrogen (Kj-N; Kjeldahl method), total ammonia nitrogen (TAN; colorimetric method, 
and un-ionized ammonia by calculation), nitrite nitro-

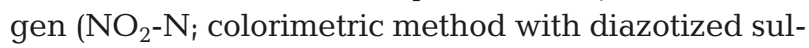
fanilamide), nitrate nitrogen $\left(\mathrm{NO}_{3}-\mathrm{N}_{i}\right.$ cadmium reduction to nitrite and measurement of nitrite), hydrogen sulfide $\left(\mathrm{H}_{2} \mathrm{~S}_{;}\right.$photometric method), total suspended solid (TSS; dried to constant weight at 103 to $\left.105^{\circ} \mathrm{C}\right)$, chlorophyll a ( $\mathrm{chl} a_{\text {; }}$ spectrophotometer method), orthophosphate $\left(\mathrm{PO}_{4}-\mathrm{P} ;\right.$ ammonium molybdate and potassium antimonyl tartrate method) and total phosphorus (TP; photometric method on sample digested by ammonium persulfate and sulfuric acid to convert all phosphorus to orthophosphate). For rainfall, the amount of rain water was measured daily by a $20 \mathrm{~cm}$ diameter Snowdon rain gauge (Mill 1907) installed next to the ponds on each farm (Fig. 1). Evaporation water was calculated for each pond through provided data from the nearest weather station.

Fish, feed and sludge. Whole fish, feed and sludge were analyzed for dry matter (DM), total $\mathrm{Kj}-\mathrm{N}$ and TP. Each sample of fish, feed and sludge was minced and homogenized. All treatment samples for TP measurements were digested by ammonium persulfate and sulfuric acid to convert all phosphorus to orthophosphate. The DM was determined gravimetrically after drying at $105^{\circ} \mathrm{C}$ for $24 \mathrm{~h}$ (AOAC 2000). Total $\mathrm{Kj}-\mathrm{N}$ was analyzed by the Kjeldahl method (AOAC 2000). The crude protein in feed was calculated from total $\mathrm{Kj}-\mathrm{N}$ multiplied by 6.25. For TP in whole fish, feed and sludge were analyzed spectrophotometrically following Kitson \& Mellon (1944). Dead fish collected during culture were weighed and counted, and the associated nutrient content was calculated based on the biweekly measured proximate composition.

The 15 fish collected from each pond at the end of the culture period were filleted manually by a professional expert from a processing company, and fillet percentage and color grade were determined according to Sang et al. (2012). The grade of fillet coloration was defined as white (score 1), pink (score 2) or yellow (score 3).

\section{Calculations and statistics}

Parameter units and calculation formulas are summarized in Table A1 in the Appendix. Water use consists only of pond-associated water use, not feedassociated water use (Verdegem et al. 2006, Verdegem \& Bosma 2009). Sludge includes the removed and accumulated fractions. Differences in water quality parameters, fish performance parameters, nutrient inputs or outputs, resource utilization parameters (consumption or use of fingerlings, water, diesel oil, electricity, labour, chemicals [lime, $\mathrm{NaCl}, \mathrm{CuSO}_{4}$ and iodine]), antibiotics, nutrients retained in fish (DM, $\mathrm{P}$, $\mathrm{N})$ and nutrient discharge (DM, P, N) between DS and US ponds were analyzed by 1-way ANOVA, followed by Tukey test in case of significant differences $(p<$ 0.05). All statistical comparisons were first done using individual weight at stocking as the co-variable. The latter was retained in the analysis when significant $(\mathrm{p}<0.05)$. Daily and biweekly measurements were averaged over the culture period before ANOVA. Daily and biweekly measurements were used to make water and nutrient mass balances (Table A1).

\section{RESULTS}

\section{Water quality}

Mean values per water quality parameter for the entire production cycle are summarized in Table 3.

Pond influent (river water)

No significant differences $(\mathrm{p}>0.05)$ were observed in pond influent between DS and US locations for $\mathrm{pH}$, TAN, ammonia nitrogen $\left(\mathrm{NH}_{3}-\mathrm{N}\right), \mathrm{NO}_{2}-\mathrm{N}_{2} \mathrm{NO}_{3}-\mathrm{N}$, $\mathrm{PO}_{4}-\mathrm{P}, \mathrm{TP}$, alkalinity, $\mathrm{CO}_{2}, \mathrm{TC}, \mathrm{TOC}, \mathrm{TSS}, \mathrm{chl}$ a, COD or $\mathrm{BOD}_{5}$ (Table 3$)$. Significant differences $(\mathrm{p}<0.05)$ were observed in pond influent water between DS and US ponds for oxygen ( $4.9 \mathrm{vs} .4 .7 \mathrm{mg} \mathrm{l}^{-1}$ ), total $\mathrm{Kj}$ $\mathrm{N}$ (3.2 vs. $6.2 \mathrm{mg} \mathrm{l}^{-1}$ ) and salinity (0.4 vs. $0.06 \mathrm{ppt}$ ).

\section{Pond water}

No significant differences $(p>0.05)$ were observed in pond water between DS and US ponds for morning $\mathrm{pH}$ and for $\mathrm{Kj}-\mathrm{N}, \mathrm{TAN}, \mathrm{NO}_{3}-\mathrm{N}, \mathrm{H}_{2} \mathrm{~S}, \mathrm{PO}_{4}-\mathrm{P}, \mathrm{CO}_{2}, \mathrm{TC}$, TOC, chl $a_{1}$ COD or $\mathrm{BOD}_{5}$. However, pond morning and afternoon water temperature, $\mathrm{NO}_{2}-\mathrm{N}, \mathrm{TP}$, alkalinity, TSS and salinity in DS ponds were significantly higher than in US ponds. The afternoon $\mathrm{pH}$, morning oxygen concentrations at 1, 2, 3 and $4 \mathrm{~m}$, afternoon oxygen concentrations at 1,2 and $3 \mathrm{~m}$, morning and afternoon water transparency, and $\mathrm{NH}_{3}-\mathrm{N}$ were lower in DS than in US ponds.

Pond influent (river water) versus pond water

There were no significant differences between DS pond influent (river water) and corresponding pond 
Table 3. Water quality in pond influent (from river) and in downstream (DS) and upstream (US) striped catfish ponds, averaged (mean \pm SD and min.-max. values) over a full production cycle and location. Chemical parameter abbreviations: Kj-N: total Kjeldahl nitrogen; TAN: total ammonia nitrogen; $\mathrm{NH}_{3}-\mathrm{N}$ : ammonia nitrogen; $\mathrm{NO}_{2}-\mathrm{N}$ : nitrite nitrogen; $\mathrm{NO}_{3}-\mathrm{N}$ : nitrate nitrogen; $\mathrm{H}_{2} \mathrm{~S}$ : hydrogen sulfide; $\mathrm{PO}_{4}-\mathrm{P}$ : orthophosphate as phosphorus; TP: total phosphorus; TC: total carbon; TOC: total organic carbon; TSS: total suspended solids; COD: chemical oxygen demand; $\mathrm{BOD}_{5}: 5 \mathrm{~d}$ biological oxygen demand. Mean values with different superscript letters in the same row are significantly different $(\mathrm{p}<0.05)$

\begin{tabular}{|c|c|c|c|c|c|c|c|c|c|}
\hline \multirow[t]{2}{*}{ Parameter } & \multicolumn{2}{|c|}{ DS influent } & \multicolumn{2}{|c|}{ DS pond } & \multicolumn{2}{|c|}{ US influent } & \multicolumn{2}{|c|}{ US pond } & \multirow{2}{*}{ p-value } \\
\hline & Mean \pm SD & Min.-max. & Mean \pm SD & Min.-max. & Mean \pm SD & Min.-max. & Mean $\pm \mathrm{SD}$ & Min.-max. & \\
\hline \multicolumn{10}{|l|}{ Temperature $\left({ }^{\circ} \mathrm{C}\right)$} \\
\hline Morning & - & - & $30.4^{\mathrm{a}} \pm 1.1$ & $28.0-33.0$ & - & - & $29.5^{\mathrm{b}} \pm 1.6$ & $27.0-33.0$ & 0.001 \\
\hline Afternoon & - & - & $31.6^{\mathrm{a}} \pm 1.3$ & $29.0-35.0$ & - & - & $30.9^{\mathrm{b}} \pm 1.2$ & $28.0-33.0$ & 0.001 \\
\hline \multicolumn{10}{|l|}{ pH } \\
\hline Morning & $6.4^{\mathrm{ab}} \pm 0.2$ & $6-7$ & $6.3^{\mathrm{c}} \pm 0.3$ & $6.0-7.0$ & $6.4^{\mathrm{a}} \pm 0.2$ & $5.7-7.0$ & $6.2^{\mathrm{c}} \pm 0.4$ & $5.6-7.2$ & 0.012 \\
\hline Afternoon & & & $6.6^{\mathrm{b}} \pm 0.4$ & $6.0-7.9$ & & & $6.7^{a} \pm 0.6$ & $5.7-7.0$ & 0.001 \\
\hline \multicolumn{10}{|l|}{ Oxygen $\left(\mathrm{mg} \mathrm{l}^{-1}\right)$} \\
\hline \multicolumn{10}{|l|}{ Morning } \\
\hline $1 \mathrm{~m}$ & & & $1.5^{\mathrm{b}} \pm 0.7$ & $0.7-3.8$ & & & $1.9^{\mathrm{a}} \pm 0.6$ & $0.6-3.6$ & 0.001 \\
\hline $2 \mathrm{~m}$ & & & $1.2^{\mathrm{d}} \pm 0.6$ & $0.5-3.4$ & & & $1.6^{\mathrm{c}} \pm 0.5$ & $0.6-3.5$ & 0.001 \\
\hline $3 \mathrm{~m}$ & & & $0.8^{\mathrm{b}} \pm 0.4$ & $0.2-2.7$ & & & $1.4^{\mathrm{a}} \pm 0.4$ & $0.5-3.4$ & 0.001 \\
\hline $3.5 \mathrm{~m}$ & $4.9^{\mathrm{a}} \pm 0.9$ & $2-6.8$ & & & $4.7^{b} \pm 1$ & $2.0-6.8$ & & & 0.001 \\
\hline $4 \mathrm{~m}$ & & & $0.5^{\mathrm{b}} \pm 0.1$ & $0.3-0.7$ & & & $1.1^{\mathrm{a}} \pm 0.4$ & $0.5-3.2$ & 0.001 \\
\hline \multicolumn{10}{|l|}{ Afternoon } \\
\hline $1 \mathrm{~m}$ & & & $1.9^{\mathrm{b}} \pm 0.1$ & $1.6-10.6$ & & & $2.2^{\mathrm{a}} \pm 0.1$ & $1.9-9.0$ & 0.001 \\
\hline $2 \mathrm{~m}$ & & & $1.5^{\mathrm{b}} \pm 0.1$ & $1.0-8.7$ & & & $1.8^{\mathrm{a}} \pm 0.1$ & $1.2-8.7$ & 0.008 \\
\hline $3 \mathrm{~m}$ & & & $1.0^{\mathrm{b}} \pm 0.1$ & $0.8-7.2$ & & & $1.3^{\mathrm{a}} \pm 0.1$ & $1.2-18.6$ & 0.001 \\
\hline $4 \mathrm{~m}$ & & & - & - & & & $0.8 \pm 0.1$ & $0.5-3.0$ & - \\
\hline \multicolumn{10}{|l|}{ Transparency (cm) } \\
\hline Morning & & & $24.6^{\mathrm{b}} \pm 6.8$ & $14.0-47.0$ & & & $30.2^{\mathrm{a}} \pm 4.4$ & $20.0-50.0$ & 0.001 \\
\hline Afternoon & & & $24.2^{\mathrm{b}} \pm 5.0$ & $15.0-48.0$ & & & $28.2^{\mathrm{a}} \pm 4.5$ & $20.0-42.0$ & 0.001 \\
\hline \multicolumn{10}{|c|}{ Chemical parameters (mg li ${ }^{-1}$, except otherwise noted) } \\
\hline $\mathrm{Kj}-\mathrm{N}$ & $3.2^{\mathrm{bc}} \pm 1.8$ & $0.5-7.3$ & $9.9^{\mathrm{a}} \pm 8.9$ & $3.0-45.7$ & $6.2^{\mathrm{ab}} \pm 3.9$ & $2.0-16.7$ & $7.1^{\mathrm{ab}} \pm 3.7$ & $1.6-22.8$ & 0.001 \\
\hline TAN & $0.19^{\mathrm{cd}} \pm 1.8$ & $0.01-0.7$ & $1.4^{\mathrm{a}} \pm 1.3$ & $0.3-5.6$ & $0.2^{\mathrm{c}} \pm 0.4$ & $0.01-1.9$ & $1.2^{\mathrm{ab}} \pm 1.1$ & $0.03-5.5$ & 0.001 \\
\hline $\mathrm{NH}_{3}-\mathrm{N}$ & $0.0^{\text {bd }}$ & $0.0-0.001$ & $0.01^{\mathrm{b}} \pm 0.0$ & $0.0-0.02$ & $0.01^{\mathrm{abc}} \pm 0.02$ & $0.0-0.7$ & $0.02^{\mathrm{a}} \pm 0.0$ & $0.0-0.1$ & 0.001 \\
\hline $\mathrm{NO}_{2}-\mathrm{N}$ & $0.02^{\mathrm{bd}} \pm 0.02$ & $0-0.7$ & $0.3^{\mathrm{a}} \pm 0.3$ & $0.0-1.4$ & $0.03^{\mathrm{bf}} \pm 0.06$ & $0.0-0.2$ & $0.1^{\mathrm{b}} \pm 0.1$ & $0.0-1.04$ & 0.001 \\
\hline $\mathrm{NO}_{3}-\mathrm{N}$ & $0.5^{\mathrm{a}} \pm 0.3$ & $0.1-1$ & $0.4^{\mathrm{a}} \pm 0.3$ & $0.3-1.0$ & $0.3^{\mathrm{a}} \pm 0.2$ & $0.1-1.0$ & $0.4^{\mathrm{a}} \pm 0.3$ & $0.02-0.5$ & 0.300 \\
\hline $\mathrm{H}_{2} \mathrm{~S}$ & - & - & $0.2^{\mathrm{a}} \pm 0.1$ & $0.01-0.5$ & - & - & $0.1^{\mathrm{a}} \pm 0.2$ & $0.0-0.8$ & 0.615 \\
\hline $\mathrm{PO}_{4}-\mathrm{P}$ & $0.04^{a} \pm 0.02$ & $0.01-0.1$ & $0.4^{\mathrm{a}} \pm 0.5$ & $0.0-1.7$ & $0.4^{\mathrm{a}} \pm 1.7$ & $0.0-7.5$ & $0.4^{\mathrm{a}} \pm 0.5$ & $0.01-1.5$ & 0.400 \\
\hline $\mathrm{TP}$ & $0.5^{\mathrm{cd}} \pm 0.3$ & $0.2-1.0$ & $2.4^{\mathrm{a}} \pm 1.2$ & $0.4-5.9$ & $0.7^{\mathrm{C}} \pm 0.3$ & $0.2-1.6$ & $1.5^{\mathrm{b}} \pm 1.0$ & $0.2-5.1$ & 0.001 \\
\hline Alkalinity & $50.7^{\mathrm{bc}} \pm 8.6$ & $40.3-65.3$ & $61.6^{\mathrm{a}} \pm 9.8$ & $48.9-83.2$ & $43.0^{\mathrm{cd}} \pm 9.2$ & $28.9-60.3$ & $51.1^{\mathrm{b}} \pm 8.7$ & $40.2-67.3$ & 0.001 \\
\hline $\mathrm{CO}_{2}$ & $34.3^{\mathrm{a}} \pm 12.8$ & $17.8-65.7$ & $42.4^{\mathrm{a}} \pm 28.1$ & $7.4-106.9$ & $28.2^{\mathrm{a}} \pm 14.2$ & $4.4-59.5$ & $30.9^{a} \pm 19.3$ & $1.2-68.3$ & 0.057 \\
\hline $\mathrm{TC}^{2}$ & $13.7^{\mathrm{d}} \pm 5.5$ & $5.6-27.3$ & $28.9^{b} \pm 16.3$ & $10.2-103.2$ & $14.8^{\mathrm{cd}} \pm 4.6$ & $5.6-27.3$ & $30.4^{\mathrm{ab}} \pm 24.1$ & $7.0-129.4$ & 0.001 \\
\hline TOC & $2.3^{\mathrm{bd}} \pm 2.2$ & $0.5-9.4$ & $8.4^{\mathrm{a}} \pm 11.5$ & $1.03-64.9$ & $3.2^{\mathrm{abc}} \pm 2.3$ & $0.8-9.4$ & $6.3^{\mathrm{ab}} \pm 5.4$ & $1.3-29.9$ & 0.015 \\
\hline TSS & $182.9^{\mathrm{ab}} \pm 47.7$ & $139.7-335.1$ & $80.8^{\mathrm{c}} \pm 33.6$ & $31.8-155.8$ & $221.3^{\mathrm{a}} \pm 51.2$ & $122.4-306.8$ & $49.3^{\mathrm{d}} \pm 21.5$ & $17.5-122$ & 0.001 \\
\hline COD & $6.0^{\mathrm{c}} \pm 1.5$ & $3.5-9.7$ & $19.4^{\mathrm{a}} \pm 5.7$ & $11.5-29.4$ & $6.9^{\mathrm{cd}} \pm 1.4$ & $4.3-8.8$ & $19.4^{\mathrm{ab}} \pm 5.1$ & $7.9-29.0$ & 0.001 \\
\hline $\mathrm{BOD}_{5}$ & $5.4^{\mathrm{c}} \pm 0.9$ & $4.2-7.2$ & $15.4^{\mathrm{a}} \pm 5.0$ & $9.1-23.7$ & $4.9^{\mathrm{cd}} \pm 1.1$ & $3.2-6.8$ & $15.0^{\mathrm{ab}} \pm 4.2$ & $7.0-24.0$ & 0.615 \\
\hline Chlorophyll a $\left(\mu \mathrm{g} \mathrm{l}^{-1}\right)$ & $6.0^{\mathrm{C}} \pm 3.2$ & $2.1-11.6$ & $114.3^{\mathrm{a}} \pm 73.5$ & $23.3-267.8$ & $5.7^{\mathrm{cd}} \pm 2.3$ & $1.9-10.2$ & $100.0^{\mathrm{ab}} \pm 45.9$ & $27.9-189.8$ & 0.001 \\
\hline Salinity (ppt) & $0.4^{\mathrm{a}} \pm 0.3$ & $0.1-1.2$ & $0.4^{\mathrm{a}} \pm 0.3$ & $0.1-1.2$ & $0.06^{b} \pm 0.02$ & $0.04-0.08$ & $0.06^{\mathrm{b}} \pm 0.02$ & $0.04-0.08$ & 0.002 \\
\hline
\end{tabular}

water for $\mathrm{NH}_{3}-\mathrm{N}, \mathrm{NO}_{3}-\mathrm{N}, \mathrm{PO}_{4}-\mathrm{P}, \mathrm{CO}_{2}$ and salinity, and between US pond influent water and corresponding pond water for $\mathrm{Kj}-\mathrm{N}, \mathrm{NH}_{3}-\mathrm{N}, \mathrm{NO}_{2}-\mathrm{N}, \mathrm{NO}_{3}-\mathrm{N}$, $\mathrm{PO}_{4}-\mathrm{P}, \mathrm{CO}_{2}$, TOC and salinity. Mean concentrations in pond influent were always lower than in the pond water for TAN, TP, alkalinity, TC, chl $a_{1} \mathrm{COD}$ and $\mathrm{BOD}_{5}$, and higher for $\mathrm{pH}$, oxygen and TSS $(\mathrm{p}<0.05)$. At DS locations, concentrations in influent were lower than in corresponding ponds for $\mathrm{Kj}-\mathrm{N}$, TAN, $\mathrm{NO}_{2}-\mathrm{N}, \mathrm{TP}$, alkalinity, TC, TOC, chl $a, \mathrm{COD}$ and $\mathrm{BOD}_{5}$. The same was observed in US ponds for TAN,
TP, alkalinity, TC, chl $a_{1}$ COD and $\mathrm{BOD}_{5}(\mathrm{p}<0.05)$. Concentrations in DS and US pond influent were similar to concentrations observed in DS and US ponds for $\mathrm{NO}_{3}-\mathrm{N}, \mathrm{PO}_{4}-\mathrm{P}$ and $\mathrm{CO}_{2}$, respectively.

\section{Growth performance}

Fish behaviour during feeding was monitored and feeding was stopped when fish began to show a decrease in appetite. In DS ponds, this resulted in real- 
ized feeding rates of $1.0 \pm 0.4,2.3 \pm 0.5$ and $2.5 \pm 0.2 \%$ body weight $\mathrm{d}^{-1}$, respectively, during culture days 1-59, 60-122, and 123 until harvest. In US ponds, realized feeding rates were $1.1 \pm 0.2,0.8 \pm$ 0.1 and $1.7 \pm 0.8 \%$ body weight $\mathrm{d}^{-1}$, respectively, during culture days 1-59, 60-122, and 123 until harvest.

In all ponds, fish were harvested when $>700 \mathrm{~g}$ average individual weight, which is considered the minimum market size. The total harvested biomass was $353 \pm 43 \mathrm{MT}$ in DS ponds and $247 \pm 21 \mathrm{MT}$ in US ponds. Total feed load was higher in DS ponds when compared with US ponds ( $p<0.05$ ). Specific growth rate was similar between DS and US ponds ( $p>0.05)$. In addition, survival, feed conversion ratio (FCR), fillet dress out percentage and fillet coloration were similar between US and DS ponds ( $\mathrm{p}>0.05)$ (Table 4).

\section{Nutrient mass balances in striped catfish ponds}

\section{Dry matter mass balance}

Feed DM input was $434 \mathrm{MT}$ in DS and $341 \mathrm{MT}$ in US ponds, of which 30 and $28 \%$ was retained as fish biomass in DS and US ponds, respectively ( $p$ > 0.05) (Table 5). Uneaten feed was likely minimal, as the provision of food was stopped at signs of low appetite during feeding. However, feed waste collectors were not deployed during or after feeding, so this is an assumption in the calculations, and we assume any feed not retained in fish biomass was therefore metabolic waste. Feeding to satiation resulted in large fluctuations in feeding between consecutive feeding days (Fig. 2). At the pond level, DM input was the sum of DM in metabolic waste and in influent water (Table 5). Mekong water provided $33 \%$ of the DM input in DS ponds and $56 \%$ in US ponds, which was significantly different $(\mathrm{p}<$ 0.05). The largest fraction of DM input was removed by pumping out sludge, which was similar between DS and US ponds (46 and $58 \%$, respectively; $\mathrm{p}>0.05$ ). Unaccounted DM was also similar between DS and US ponds (37 and $29 \%$, respectively; $p>0.05$ ).
Table 4. Fish performance and feeding in downstream (DS) and upstream (US) striped catfish ponds. Values are means $\pm \mathrm{SD}(\mathrm{n}=2)$. BW: body weight; MT: metric tonnes; WW: wet weight. $\left({ }^{*}\right)$ : parameters with a significant $(\mathrm{p}<0.05$, not shown) co-variable effect of individual weight at stocking. Period 1: Day 1-59; Period 2: Day 60-122; Period 3: Day 123-harvest

\begin{tabular}{|c|c|c|c|}
\hline Parameter & DS pond & US pond & $\mathrm{p}$-value \\
\hline Final BW (g ind.$^{-1}$ ) & $791.00 \pm 14.1$ & $875.00 \pm 63.6$ & 0.210 \\
\hline Initial number of fish $\left(\times 10^{3}\right)$ & $600.00 \pm 183.8$ & $553.50 \pm 1.4$ & 0.755 \\
\hline Initial biomass* (MT) & $27.40 \pm 7.9$ & $15.70 \pm 0.4$ & 0.171 \\
\hline Final biomass (MT) & $352.80 \pm 43.1$ & $247.10 \pm 21.4$ & 0.090 \\
\hline Yield ( $\mathrm{kg}$ fish $\mathrm{m}^{-2}$ ) & $31.80 \pm 3.9$ & $22.90 \pm 2.0$ & 0.117 \\
\hline Yield ( $\mathrm{kg}$ fish $\mathrm{m}^{-3}$ ) & $9.30 \pm 0.4$ & $5.50 \pm 1.2$ & 0.054 \\
\hline Total feed (MT, WW) & $486.40 \pm 15.0$ & $381.00 \pm 28.8$ & 0.044 \\
\hline Survival $^{*}(\%)$ & $76.40 \pm 13.0$ & $51.00 \pm 0.6$ & 0.110 \\
\hline Specific growth rate $\left(\% \mathrm{BW} \mathrm{d}^{-1}\right)$ & $1.24 \pm 0.0$ & $1.21 \pm 0.4$ & 0.746 \\
\hline Period $1\left(\% \mathrm{BW} \mathrm{d}^{-1}\right)$ & $0.82 \pm 0.2$ & $0.95 \pm 0.2$ & 0.575 \\
\hline Period $2\left(\% \mathrm{BW} \mathrm{d}^{-1}\right)$ & $1.19 \pm 0.6$ & $1.34 \pm 0.2$ & 0.764 \\
\hline Period $3\left(\% \mathrm{BW} \mathrm{d}^{-1}\right)$ & $1.45 \pm 0.1$ & $1.24 \pm 0.1$ & 0.191 \\
\hline Feed conversion ratio & $1.50 \pm 0.1$ & $1.65 \pm 0.0$ & 0.211 \\
\hline Fillet percentage (\%) & $35.50 \pm 1.2$ & $35.20 \pm 1.7$ & 0.465 \\
\hline Fillet colour grade (1-3) & $1.47 \pm 0.6$ & $1.20 \pm 0.5$ & 0.071 \\
\hline
\end{tabular}

Table 5. Dry matter (DM) mass balance in downstream (DS) and upstream (US) striped catfish ponds per production cycle. DM in feed not retained in live or dead fish is considered to become metabolic waste. Values (in $10^{3} \mathrm{~kg}$ ) are means $\pm \mathrm{SD}(\mathrm{n}=2)$

\begin{tabular}{|lcrcrr|}
\hline $\begin{array}{l}\text { Mass balance } \\
\text { component }\end{array}$ & \multicolumn{2}{c}{ DS pond } & \multicolumn{2}{c}{ US pond } & p-value \\
& Mean \pm SD & $\%$ & Mean \pm SD & $\%$ & \\
Fish level & & & & & \\
Feed & $434.0 \pm 13.4$ & 100.0 & $340.6 \pm 25.8$ & 100.0 & 0.045 \\
Live fish & $123.7 \pm 14.0$ & 28.5 & $88.1 \pm 8.6$ & 25.9 & 0.092 \\
Dead fish & $4.7 \pm 2.1$ & 1.1 & $6.2 \pm 0.9$ & 1.8 & 0.415 \\
Metabolic waste & $305.6 \pm 2.8$ & 70.4 & $246.3 \pm 17.1$ & 72.3 & 0.040 \\
& & & & & \\
Pond level & & & & & \\
DM input & & & & & \\
$\quad$ Metabolic waste & $305.6 \pm 2.8$ & 67.3 & $246.3 \pm 17.1$ & 44.1 & 0.040 \\
Influent & $148.3 \pm 13.1$ & 32.7 & $311.6 \pm 11.1$ & 55.9 & 0.006 \\
$\quad$ Total & $453.9 \pm 10.4$ & 100.0 & $557.9 \pm 28.2$ & 100.0 & 0.039 \\
DM output & & & & & \\
$\quad$ Effluent & $74.5 \pm 5.9$ & 16.4 & $68.7 \pm 0.5$ & 12.3 & 0.295 \\
Sludge & $209.5 \pm 35.7$ & 46.2 & $325.6 \pm 22.6$ & 58.4 & 0.060 \\
& & & & & \\
Unaccounted & $169.9 \pm 31.3$ & 37.4 & $163.6 \pm 5.1$ & 29.3 & 0.806 \\
\hline
\end{tabular}

P mass balance

More $\mathrm{P}$ was administrated with the feed in DS ponds than in US ponds ( $p<0.05$ ) (Table 6). On average, $18 \%$ of feed-P was retained in fish biomass, including dead fish, in both DS and US ponds. The remaining $82 \%$ became metabolic waste. Of the combined $\mathrm{P}$ input through metabolic waste and river influent water, $34 \%$ in DS ponds and 38\% in US ponds was discharged with effluent water. In the P mass balance, 


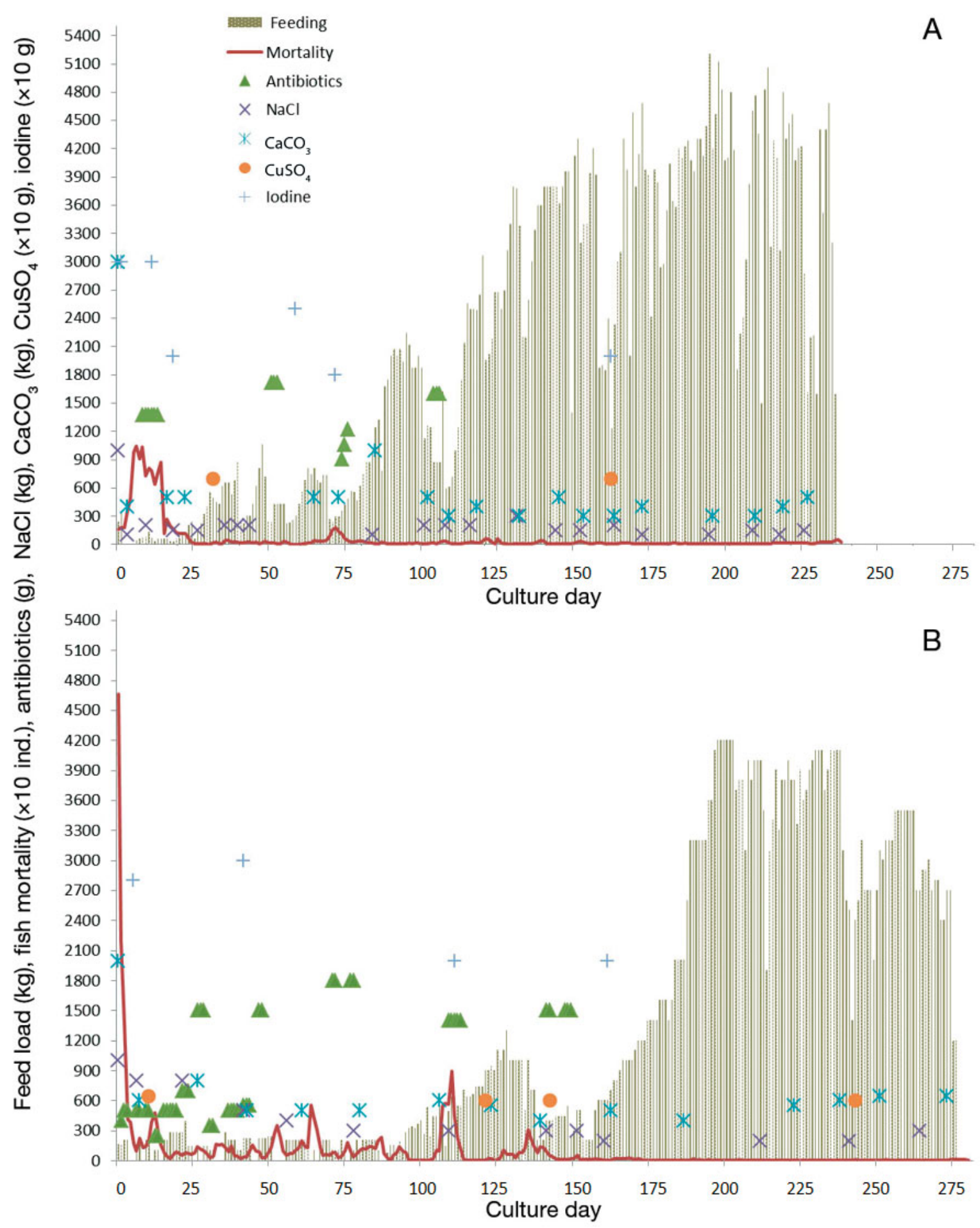

Fig. 2. Daily feed load, fish mortality, and antibiotic and chemical use during the culture period in (A) downstream (DS) and (B) upstream (US) ponds

0.8 and $2.1 \%$ remained unaccounted in DS and US ponds, respectively, at the end of the production cycle. The largest fraction of $\mathrm{P}$ was removed with sludge pumped out of the pond ( $65 \%$ of metabolic waste in DS and $59 \%$ in US ponds; $\mathrm{p}>0.05$ ).
$\mathrm{N}$ mass balance

More $\mathrm{N}$ was administrated with the feed in DS ponds than in US ponds $(p<0.05)$ (Table 7). The percentage of feed-N retained in fish, including dead 
Table 6. Phosphorus (P) mass balance in downstream (DS) and upstream (US) striped catfish ponds per production cycle. For details on fish level components, see Table 5. Values (in $\left.10^{3} \mathrm{~kg}\right)$ are means $\pm \mathrm{SD}(\mathrm{n}=2)$

\begin{tabular}{|c|c|c|c|c|c|}
\hline $\begin{array}{l}\text { Mass balance } \\
\text { component }\end{array}$ & \multicolumn{2}{|c|}{ DS pond } & \multicolumn{2}{|c|}{ US pond } & $\mathrm{p}$-value \\
\hline \multicolumn{6}{|l|}{ Fish level } \\
\hline Feed & $6.73 \pm 0.20$ & 100.0 & $5.11 \pm 0.38$ & 100.0 & 0.034 \\
\hline Live fish & $1.09 \pm 0.11$ & 16.2 & $0.77 \pm 0.88$ & 15.1 & 0.082 \\
\hline Dead fish & $0.10 \pm 0.03$ & 1.5 & $0.13 \pm 0.06$ & 2.5 & 0.261 \\
\hline Metabolic waste & $5.54 \pm 0.07$ & 82.5 & $4.20 \pm 0.30$ & 82.2 & 0.025 \\
\hline \multicolumn{6}{|l|}{ Pond level } \\
\hline \multicolumn{6}{|l|}{ P input } \\
\hline Metabolic waste & $5.54 \pm 0.07$ & 92.3 & $4.20 \pm 0.30$ & 81.9 & 0.025 \\
\hline Influent & $0.46 \pm 0.01$ & 7.7 & $0.93 \pm 0.16$ & 18.1 & 0.055 \\
\hline Total & $6.00 \pm 0.07$ & 100.0 & $5.13 \pm 0.46$ & 100.0 & 0.119 \\
\hline \multicolumn{6}{|l|}{ P output } \\
\hline Effluent & $2.05 \pm 0.27$ & 34.2 & $1.96 \pm 0.20$ & 38.2 & 0.746 \\
\hline Sludge & $3.90 \pm 0.18$ & 65.0 & $3.05 \pm 0.30$ & 59.2 & 0.075 \\
\hline Unaccounted & $0.05 \pm 0.01$ & 0.8 & $0.11 \pm 0.04$ & 2.1 & 0.152 \\
\hline
\end{tabular}

Table 7. Nitrogen (N) mass balance in downstream (DS) and upstream (US) striped catfish ponds per production cycle. For details on fish level components, see Table 5. Values (in $\left.10^{3} \mathrm{~kg}\right)$ are means $\pm \mathrm{SD}(\mathrm{n}=2)$

\begin{tabular}{|c|c|c|c|c|c|}
\hline \multirow{2}{*}{$\begin{array}{l}\text { Mass balance } \\
\text { component }\end{array}$} & \multicolumn{2}{|c|}{ DS pond } & \multicolumn{2}{|c|}{ US pond } & \multirow[t]{2}{*}{$\mathrm{p}$-value } \\
\hline & Mean \pm SD & $\%$ & Mean \pm SD & $\%$ & \\
\hline \multicolumn{6}{|l|}{ Fish level } \\
\hline Feed & $20.78 \pm 0.69$ & 100.0 & $16.13 \pm 1.22$ & 100.0 & 0.043 \\
\hline Live fish & $8.22 \pm 0.84$ & 39.6 & $5.88 \pm 0.58$ & 36.5 & 0.082 \\
\hline Dead fish & $0.96 \pm 0.67$ & 4.6 & $0.50 \pm 0.02$ & 3.1 & 0.441 \\
\hline Metabolic waste & $11.60 \pm 0.52$ & 55.8 & $9.76 \pm 0.67$ & 60.4 & 0.092 \\
\hline \multicolumn{6}{|l|}{ Pond level } \\
\hline \multicolumn{6}{|l|}{$\mathrm{N}$ input } \\
\hline Metabolic waste & $11.60 \pm 0.52$ & 77.0 & $9.76 \pm 0.67$ & 57.3 & 0.092 \\
\hline Influent & $3.47 \pm 0.35$ & 23.0 & $7.27 \pm 0.55$ & 42.7 & 0.014 \\
\hline Total & $15.07 \pm 0.87$ & 100.0 & $17.03 \pm 0.12$ & 100.0 & 0.088 \\
\hline \multicolumn{6}{|l|}{ N output } \\
\hline Effluent & $8.51 \pm 1.17$ & 56.5 & $10.0 \pm 0.15$ & 58.7 & 0.215 \\
\hline Sludge & $1.42 \pm 0.91$ & 9.4 & $1.89 \pm 0.22$ & 11.1 & 0.557 \\
\hline Unaccounted & $5.139 \pm 1.12$ & 34.1 & $5.15 \pm 0.21$ & 30.2 & 0.991 \\
\hline
\end{tabular}

fish, was similar in DS and US ponds, fluctuating between 39 and $44 \%$ ( $p>0.05)$. The remaining $56-60 \%$ of feed-N became metabolic waste. Of the combined $\mathrm{N}$ input through metabolic waste and influent water, $57-59 \%$ was discharged with effluent water, 9-11\% pumped out with sludge, and $30-34 \%$ remained unaccounted. These results were similar for DS and US ponds $(p>0.05)$. Assuming the unaccounted $N$ volatilized, then in DS ponds $44 \%$ of the metabolic waste volatilized while in US ponds $53 \%$ volatilized. As a consequence, 56 and $47 \%$ of the metabolic waste was discharged to the Mekong River from DS and US ponds, respectively.

\section{Pond management}

Pond sustainability indicators are presented in Table 8. Use of water, electricity, labour, lime, salt and antibiotics $\mathrm{kg}^{-1}$ fish produced were 61,71 , $45,66,49$ and $60 \%$ lower, respectively, in DS than in US ponds $(\mathrm{p}<0.05)$. However, diesel oil use and sludge removal frequency $\mathrm{kg}^{-1}$ fish produced were, respectively, 113 and $200 \%$ higher in DS ponds than in US ponds $(p<0.05)$. No differences between DS and US ponds were observed for $\mathrm{CuSO}_{4}$ or iodine use $(\mathrm{p}>0.05)$. The period of no antibiotic use before harvest was $133 \mathrm{~d}$ in DS ponds and $126 \mathrm{~d}$ in US ponds (Fig. 2).

\section{DISCUSSION}

\section{Pond performance}

None of the measured water quality parameters in the DS or US pond influent exceeded the limits set by the Vietnamese Ministry of Agriculture and Rural Development for striped catfish pond farming (MARD 2003). The FCR over the full production cycle was on average 1.5 in DS ponds and 1.7 in US ponds, in agreement with earlier reported FCRs for striped catfish farming in the Mekong Delta (Bosma et al. 2009, Phan et al. 2009). Observed differences in survival between DS and US ponds were possibly due to differences in management and water regimes at the 2 locations. In all ponds, the fraction of input-P remaining unexplained over the complete production cycle was less than $-2 \%$, which is lower than reported by Adhikari et al. (2014) and Thakur \& Lin (2003). A higher fraction of DM and N remained unexplained in the mass balance, because contrary to $\mathrm{P}$, not all possible sinks were measured. It is assumed that the unaccounted fractions of $\mathrm{DM}$ and $\mathrm{N}$ in the mass balances were mainly volatilized as $\mathrm{CO}_{2}$ and $\mathrm{N}_{2}$, respectively (Boyd 1985, Lin et al. 1997, Funge- 
Table 8. Sustainability indicator expressed per $\mathrm{kg}$ feed (based on wet weight) consumed and per kg fish produced for downstream (DS) and upstream (US) striped catfish ponds. Values are means \pm SD (n = 2); DM: dry matter

\begin{tabular}{|c|c|c|c|c|c|c|}
\hline \multirow{2}{*}{ Parameter } & \multirow{2}{*}{ Unit } & \multicolumn{2}{|c|}{ DS pond } & \multicolumn{2}{|c|}{ US pond } & \multirow[t]{2}{*}{$\mathrm{p}$-value } \\
\hline & & Mean $\pm \mathrm{SD}$ & Min.-max. & Mean \pm SD & Min.-max. & \\
\hline \multicolumn{7}{|l|}{ Resource use } \\
\hline Fingerlings use & No. $\mathrm{kg}^{-1}$ fish & $1.70 \pm 0.30$ & $1.50-1.90$ & $2.20 \pm 0.20$ & $2.10-2.40$ & 0.161 \\
\hline \multirow[t]{2}{*}{ Water use } & $\mathrm{m}^{3} \mathrm{~kg}^{-1}$ feed & $1.90 \pm 0.10$ & $1.80-1.90$ & $4.30 \pm 0.10$ & $4.30-4.40$ & 0.001 \\
\hline & $\mathrm{m}^{3} \mathrm{~kg}^{-1}$ fish & $2.80 \pm 0.10$ & $2.70-2.90$ & $7.10 \pm 0.0$ & $7.12-7.14$ & 0.001 \\
\hline \multirow[t]{2}{*}{ Diesel oil use } & $\mathrm{ml} \mathrm{kg}{ }^{-1}$ feed & $1.10 \pm 0.10$ & $1.10-1.20$ & $0.50 \pm 0.01$ & $0.40-0.60$ & 0.013 \\
\hline & $\mathrm{ml} \mathrm{kg}{ }^{-1}$ fish & $1.60 \pm 0.30$ & $1.40-1.80$ & $0.75 \pm 0.03$ & $0.70-0.80$ & 0.048 \\
\hline \multirow[t]{2}{*}{ Electricity use } & $\mathrm{kWh} \mathrm{kg}^{-1}$ feed & $0.02 \pm 0.01$ & $0.02-0.03$ & $0.09 \pm 0.01$ & $0.08-1.00$ & 0.007 \\
\hline & $\mathrm{kWh} \mathrm{kg}^{-1}$ fish & $0.04 \pm 0.01$ & $0.03-0.04$ & $0.14 \pm 0.01$ & $0.10-0.15$ & 0.004 \\
\hline \multirow[t]{2}{*}{ Labour use } & $\mathrm{h} \mathrm{kg}^{-1}$ feed & $0.05 \pm 0.00$ & $0.046-0.05$ & $0.07 \pm 0.01$ & $0.06-0.07$ & 0.030 \\
\hline & $\mathrm{h} \mathrm{kg}^{-1}$ fish & $0.06 \pm 0.00$ & $0.050-0.07$ & $0.11 \pm 0.01$ & $0.10-0.12$ & 0.035 \\
\hline \multicolumn{7}{|l|}{ Chemical use } \\
\hline \multirow[t]{2}{*}{ Lime $\left(\mathrm{CaCO}_{3}\right)$} & $\mathrm{g} \mathrm{kg}^{-1}$ feed & $8.50 \pm 0.30$ & $8.30-8.70$ & $25.40 \pm 2.70$ & $23.50-27.30$ & 0.013 \\
\hline & $\mathrm{g} \mathrm{kg}^{-1}$ fish & $14.40 \pm 3.60$ & $11.80-16.90$ & $41.90 \pm 5.10$ & $38.30-45.50$ & 0.025 \\
\hline \multirow[t]{2}{*}{ Salt $(\mathrm{NaCl})$} & $\mathrm{g} \mathrm{kg}^{-1}$ feed & $7.10 \pm 0.60$ & $6.60-7.40$ & $12.00 \pm 0.90$ & $11.40-12.60$ & 0.023 \\
\hline & $\mathrm{g} \mathrm{kg}^{-1}$ fish & $10.60 \pm 1.70$ & $9.40-11.80$ & $20.90 \pm 0.30$ & $20.70-21.10$ & 0.014 \\
\hline \multirow[t]{2}{*}{$\mathrm{CuSO}_{4}$} & $\mathrm{~g} \mathrm{~kg}^{-1}$ feed & $0.03 \pm 0.02$ & $0.01-0.04$ & $0.04 \pm 0.01$ & $0.03-0.06$ & 0.235 \\
\hline & $\mathrm{g} \mathrm{kg}^{-1}$ fish & $0.04 \pm 0.02$ & $0.02-0.05$ & $0.09 \pm 0.01$ & $0.06-0.11$ & 0.226 \\
\hline \multirow[t]{2}{*}{ Iodine } & $\mathrm{g} \mathrm{kg}^{-1}$ feed & $0.16 \pm 0.06$ & $0.12-0.20$ & $0.20 \pm 0.12$ & $0.20-0.21$ & 0.173 \\
\hline & $\mathrm{g} \mathrm{kg}^{-1}$ fish & $0.30 \pm 0.08$ & $0.17-0.33$ & $0.40 \pm 0.01$ & $0.04-0.43$ & 0.165 \\
\hline \multirow[t]{2}{*}{ Antibiotic use } & $\mathrm{g} \mathrm{kg}^{-1}$ feed & $0.04 \pm 0.01$ & $0.03-0.05$ & $0.09 \pm 0.01$ & $0.08-0.97$ & 0.020 \\
\hline & $\mathrm{g} \mathrm{kg}^{-1}$ fish & $0.06 \pm 0.01$ & $0.05-0.07$ & $0.15 \pm 0.01$ & $0.14-0.16$ & 0.020 \\
\hline \multicolumn{7}{|c|}{ Nutrient use efficiency } \\
\hline DM retained & $\%$ & $29.5 \pm 2.8$ & $27.5-31.5$ & $27.7 \pm 0.5$ & $27.3-28.0$ & 0.451 \\
\hline $\mathrm{N}$ retained & $\%$ & $44.2 \pm 0.7$ & $43.7-44.6$ & $39.5 \pm 0.4$ & $39.2-39.8$ & 0.014 \\
\hline$P$ retained & $\%$ & $17.7 \pm 1.4$ & $16.7-18.7$ & $17.6 \pm 0.4$ & $17.3-17.9$ & 0.973 \\
\hline \multicolumn{7}{|c|}{ Nutrient discharge } \\
\hline \multirow[t]{2}{*}{ DM discharge } & $\mathrm{g} \mathrm{kg}^{-1}$ fish & $414.7 \pm 42.0$ & $384.6-444.9$ & $356.5 \pm 19.3$ & $342.8-370.2$ & 0.221 \\
\hline & $\mathrm{g} \mathrm{kg}^{-1}$ feed & $278.2 \pm 49.9$ & $242.9-313.6$ & $216.5 \pm 14.9$ & $205.9-227.1$ & 0.236 \\
\hline \multirow[t]{2}{*}{$\mathrm{N}$ discharge } & $\mathrm{g} \mathrm{kg}^{-1}$ fish & $19.8 \pm 2.0$ & $17.2-22.9$ & $20.1 \pm 4.0$ & $18.4-21.2$ & 0.943 \\
\hline & $\mathrm{g} \mathrm{kg}^{-1}$ feed & $13.3 \pm 1.7$ & $12.1-14.5$ & $12.0 \pm 1.4$ & $11.1-13.0$ & 0.493 \\
\hline \multirow{2}{*}{ P discharge } & $\mathrm{g} \mathrm{kg}^{-1}$ fish & $17.0 \pm 1.6$ & $15.8-18.1$ & $17.7 \pm 0.2$ & $17.6-17.8$ & 0.579 \\
\hline & $\mathrm{g} \mathrm{kg}^{-1}$ feed & $11.3 \pm 0.2$ & $11.2-11.4$ & $10.7 \pm 0.1$ & $10.7-10.8$ & 0.054 \\
\hline
\end{tabular}

Smith \& Briggs 1998, Gross et al. 2000, Adhikari et al. 2014), and that other fractions in the mass balances were quantified with the same accuracy as for P.

\section{Water exchange and water quality}

Water exchange was one of the main factors influencing pond water quality. Water use in US ponds was $7.1 \mathrm{~m}^{3} \mathrm{~kg}^{-1}$ fish, which was 2.5 times higher than the $2.8 \mathrm{~m}^{3} \mathrm{~kg}^{-1}$ fish exchanged in DS ponds. Higher daily water exchange with the Mekong River in US ponds concurred with significantly lower pond concentrations for $\mathrm{NO}_{2}-\mathrm{N}$, TP, alkalinity and $\mathrm{CO}_{2}$ compared to DS ponds. The observed difference in $\mathrm{Kj}-\mathrm{N}$ concentration of $3.2 \mathrm{mg} \mathrm{l}^{-1}$ in influent in DS ponds compared to $6.2 \mathrm{mg} \mathrm{l}^{-1}$ in US pond influent resulted in $16 \%$ addi- tional protein load $\mathrm{kg}^{-1}$ feed in US ponds. Considering $\mathrm{Kj}-\mathrm{N}$ concentrations in influent water, an estimated $58 \mathrm{~g}$ additional protein (13\%) $\mathrm{kg}^{-1}$ feed entered DS ponds and $161 \mathrm{~g}(58 \%) \mathrm{kg}^{-1}$ feed entered US ponds. This might-depending on settling properties, inpond water retention time and degradability — impact oxygen availability for fish and microbial respiration. If completely decomposed and not accounting for oxidation of nitrogen, $1 \mathrm{~g}$ protein consumes $1.77 \mathrm{~g}$ oxygen (Dalsgaard \& Pedersen 2011), which indicates an additional oxygen demand of 102 and $285 \mathrm{~g} \mathrm{O}_{2} \mathrm{~kg}^{-1}$ feed in DS and US ponds, respectively. If only $10 \%$ of this protein were degraded in the pond it means 35 and 59\% more oxygen demand in DS and US ponds, respectively, would be required than the amount supplied by the influent water. Hence, the exchange water significantly increased pond oxygen demand. 
The low difference in oxygen concentration of $4.9 \mathrm{mg} \mathrm{l}^{-1}$ in influent water to DS ponds versus $4.7 \mathrm{mg}$ $\mathrm{l}^{-1}$ to US ponds had a negligible effect on striped catfish growth performance. During the production cycle, growth performance remained favourable, even with dissolved oxygen dropping below $2 \mathrm{mg} \mathrm{O}_{2}$ $\mathrm{l}^{-1}$ at $1 \mathrm{~m}$ depth within $2 \mathrm{mo}$ after stocking. This is possible because striped catfish are facultative airbreathers (Lefevre et al. 2011b), and therefore dissolved oxygen in the water is less important for maintaining growth. To indicate this, we assumed a conservative oxygen consumption of $200 \mathrm{~g} \mathrm{O}_{2} \mathrm{~kg}^{-1}$ feed intake by striped catfish (Table 9), similar to the air-breathing African catfish Clarias gariepinus (Eding \& Weerd 1999). This conforms with a feedbased oxygen consumption of $37.4 \mathrm{~g} \mathrm{O}_{2} \mathrm{~m}^{-2} \mathrm{~d}^{-1}$, concurring with an average feed load of $187 \mathrm{~g}$ feed $\mathrm{m}^{-2}$ $\mathrm{d}^{-1}$ in both DS and US ponds. For the pond culture conditions in the current study, the oxygen supply was estimated to be $0.96 \mathrm{~g} \mathrm{O}_{2} \mathrm{~m}^{-2} \mathrm{~d}^{-1}$ through water exchange, $21.5 \mathrm{~g} \mathrm{O}_{2} \mathrm{~m}^{-2} \mathrm{~d}^{-1}$ through primary production and $3 \mathrm{~g} \mathrm{O}_{2} \mathrm{~m}^{-2} \mathrm{~d}^{-1}$ through the water surface oxygen exchange. Oxygen consumption in the water column was estimated at $10.47 \mathrm{~g} \mathrm{O}_{2} \mathrm{~m}^{-2} \mathrm{~d}^{-1}$ and $12.42 \mathrm{~g} \mathrm{O}_{2} \mathrm{~m}^{-2} \mathrm{~d}^{-1}$ in the sediment. This leads to an oxygen deficit of $34.8 \mathrm{~g} \mathrm{O}_{2} \mathrm{~m}^{-2} \mathrm{~d}^{-1}$, equaling $93 \%$ $(34.8 / 37.4 \times 100)$ of the oxygen requirement of striped catfish in the pond (Table 9). This in part explains why the fish remained close to the surface, even in deep ponds (Lefevre et al. 2011a). Striped catfish prefer, under non-fed conditions, to consume oxygen from the water column provided there is suf- ficient oxygen (Lefevre et al. 2011b). However, under fed conditions, results show that striped catfish in the Mekong Delta are produced under dissolved oxygen deficient conditions. This observation means that striped catfish can be produced under low water exchange conditions, because only $7 \%$ of the oxygen demand $\mathrm{kg}^{-1}$ feed ( $=14 \mathrm{~g} \mathrm{O}_{2} \mathrm{~kg}^{-1}$ feed) was provided by dissolved oxygen resources. Assuming surface aeration is negligible, at $30^{\circ} \mathrm{C}$ a flow rate of 7 to $8 \mathrm{~m}^{3}$ $\mathrm{kg}^{-1}$ feed $\mathrm{d}^{-1}$ should be sufficient to cover the water oxygen demand of striped catfish (influent concentration $7.2 \mathrm{mg} \mathrm{O}_{2} \mathrm{l}^{-1}$ [95\% saturated], effluent $4.5 \mathrm{mg}$ $\mathrm{O}_{2} \mathrm{l}^{-1}$ ). A higher flow rate of $12 \mathrm{~m}^{3} \mathrm{~kg}^{-1}$ feed was reported for African catfish by Eding \& Kamstra (2002) to control oxygen and ammonia and assuming the fish consumed $300 \mathrm{~g} \mathrm{O}_{2} \mathrm{~kg}^{-1}$ feed.

The average $\mathrm{H}_{2} \mathrm{~S}$ concentration was $0.1-0.2 \mathrm{mg} \mathrm{l}^{-1}$, and was similar in DS and US ponds, with peak concentrations reaching $0.5-0.8 \mathrm{mg} \mathrm{l}^{-1}$ (Table 3). Considering the $\mathrm{NO}_{3}-\mathrm{N}$ concentrations in the ponds were low $\left(0.3-0.5 \mathrm{mg} \mathrm{l}^{-1}\right)$, diffusion of $\mathrm{NO}_{3}-\mathrm{N}$ into the flocculent bottom layer was too low to prevent $\mathrm{H}_{2} \mathrm{~S}$ formation under oxygen depleted conditions. The large water volume and daily exchange in the ponds helped to keep $\mathrm{H}_{2} \mathrm{~S}$ concentrations low. The sludge removal frequency (3 times in DS ponds, 1 time in US ponds) was sufficient to maintain favourable water quality, as in our study no correlation was found between fish growth or fish mortality and $\mathrm{H}_{2} \mathrm{~S}$ concentration ( $p>0.05)$. Nevertheless, the $\mathrm{H}_{2} \mathrm{~S}$ concentration increased during culture $\left(\mathrm{H}_{2} \mathrm{~S}\left[\mathrm{mg} \mathrm{l}^{-1}\right]=\right.$ $0.0016 \times$ culture day $-0.0192 ; \mathrm{r}^{2}=0.5649, \mathrm{p}<0.05$ )

Table 9. Estimated oxygen mass balance of an average downstream pond, based on average water quality, feed input and fish biomass considering the complete production cycle. BOD $_{5}$ : 5 d biological oxygen demand; DM: dry matter; DO: dissolved oxygen

\begin{tabular}{|c|c|c|c|c|c|}
\hline \multirow[t]{2}{*}{ Parameter } & \multicolumn{2}{|c|}{$\mathrm{g} \mathrm{O}_{2} \mathrm{~m}^{-2} \mathrm{~d}^{-1}$} & \multicolumn{2}{|c|}{$\%$ of fish respiration } & \multirow[t]{2}{*}{ Reference } \\
\hline & Input & Sink & Input & Sink & \\
\hline 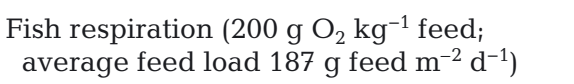 & & 37.40 & & 100.0 & Eding \& Weerd (1999) \\
\hline $\begin{array}{l}\text { Water exchange } \\
\left(0.36 \mathrm{~m}^{3} \mathrm{~m}^{-2}, 3.7 \mathrm{~g} \mathrm{O}_{2} \mathrm{~m}^{-3} \mathrm{~d}^{-1}\right)\end{array}$ & 1.32 & & 3.52 & & Present study \\
\hline $\begin{array}{l}\text { Water column respiration } \\
\text { (based on } \mathrm{BOD}_{5} \text { measurement) }\end{array}$ & & 10.47 & & 28.0 & Present study \\
\hline $\begin{array}{l}\text { Gross photosynthesis (assuming } 3 \% \\
\text { chl } a \text { in algae } \mathrm{DM} 3.47 \mathrm{~g} \mathrm{O}_{2} \mathrm{~g}^{-1} \mathrm{C} \text { fixed) }\end{array}$ & 21.51 & & 57.05 & & $\begin{array}{l}\text { Desortová (1981), } \\
\text { Drapcho \& Brune (2000) }\end{array}$ \\
\hline $\begin{array}{l}\text { Surface gas exchange } \\
\left(2 \mathrm{~m} \mathrm{~s}^{-1} \text { wind speed at } 30^{\circ} \mathrm{C}\right)\end{array}$ & 3.00 & & 8.00 & & Boyd \& Tucker (1998) \\
\hline $\begin{array}{l}\text { Sediment oxygen demand } \\
\text { (based on } \mathrm{BOD}_{5} \text { measurement) }\end{array}$ & & 12.42 & & 33.20 & Present study \\
\hline DO deficit & \multicolumn{2}{|c|}{34.46} & \multicolumn{2}{|c|}{92.15} & Present study \\
\hline
\end{tabular}


and was similar between DS and US locations ( $\mathrm{p}>$ 0.05). Furthermore, the observed $\mathrm{H}_{2} \mathrm{~S}$ concentrations always remained below threshold concentrations reported to affect fish growth and fillet meat quality (Linh 2012). $\mathrm{H}_{2} \mathrm{~S}$ concentrations were measured biweekly, and occasionally reached a peak value of $0.8 \mathrm{mg} \mathrm{l}^{-1}$. Linh (2012) reported a $10 \%$ lethal concentration $\left(\mathrm{LC}_{10}\right)$ for striped catfish of $0.83 \mathrm{mg} \mathrm{l}^{-1}$ over a $60 \mathrm{~d}$ observation period. Daily monitoring of $\mathrm{H}_{2} \mathrm{~S}$ concentrations and investigating possible chronic effects of low $\mathrm{H}_{2} \mathrm{~S}$ on striped catfish survival over the full production cycle requires further research.

The $\mathrm{NO}_{2}-\mathrm{N}$ concentration was never higher than $1 \mathrm{mg} \mathrm{l}^{-1}$, which is much lower than reported safe levels for striped catfish (Huong et al. 2011). Salinity of influent water to DS ponds (0.4 ppt; range: $0.1-1.2$ ) was higher than in US ponds (0.06 $\mathrm{ppt}_{\text {; }}$ range: $0.04-0.08)$. These parameters did not influence survival or fish yield, which were similar between DS and US ponds ( $p=0.11$ and 0.12 , respectively).

\section{Water quality maintenance for fish growth}

The FCR over the full production cycle was on average 1.5 in DS ponds and 1.7 in US ponds, which corresponds with earlier reported FCRs for striped catfish farming in the Mekong Delta (Bosma et al. 2009, Phan et al. 2009). The fact that the specific growth rate (SGR) increased in DS ponds during the production cycle (Table 4) is contradictory to normal culture conditions for other fish species, including striped catfish. Temperature remained close to optimal during the culture period, and cannot explain the increase in SGR during culture. Fish mortality due to disease was high during period 1 (Days 1-59), and when disease occurred, feeding was reduced or even suspended. Ponds were not fed 15 and $17 \%$ of culture days in DS and US ponds, respectively (Fig. 2A,B). The larger feed input deficiency in US ponds concurred with a culture period of $277 \mathrm{~d}$ compared to $234 \mathrm{~d}$ in DS ponds. A $43 \mathrm{~d}$ longer culture period in US ponds required more labour and energy, delayed income from fish sales and increased emissions of chemicals and drugs. In US ponds, the longer culture period concurred with reduced feeding during disease treatment, either by application of antibiotics or $\mathrm{CuSO}_{4}$ (Fig. 2A,B). Improving biosecurity, stocking disease-free fingerlings, controlling pathogens (including parasites) in the exchange water (Phan et al. 2009) and reducing transmission by possible hosts (Bondad-Reantaso et al. 2005) would contri- bute to better growth and feed utilization. Disease control in striped catfish pond culture remains a challenge, considering it is an open system connected to the Mekong River.

Striped catfish farmers prefer 1 ha ponds that are 2 to $6 \mathrm{~m}$ deep, located along channels and rivers accessible by ships to supply feed and transport harvested fish to the processing plant (Phan et al. 2009). The culture cycle starts with 2-3 m water depth during the first month, which is subsequently raised to the maximum pond depth and maintained until the end of the production cycle. In deep ponds, water movement above the bottom is minimal, resulting in negligible resuspension of settled sludge. Fish-driven resuspension is also negligible as the fish stay close to the surface. The farmer checks the height of the sludge layer above the sediment, and removes it when it becomes too high. Water exchange pipes are situated at $\pm 2 \mathrm{~m}$ depth, allowing the exchange of large volumes of surface water with minimum disturbance to the deep-water layers in the pond (especially the flocculent layer) to avoid fish gill damage. The decision to culture striped catfish in 4-6 m deep ponds developed as an adaptation to the semi-tidal conditions in the Mekong Delta with two $3 \mathrm{~h}$ tidal water exchange periods daily, allowing use of the first period to discharge and the second period to take in water. As a consequence, between tides, the pond water volume is lower than when the pond is full. By using deep ponds, the risks of resuspension and a high suspended solid concentration in the water column is reduced during the period of low water levels. When too much sludge accumulates at the bottom, it is pumped to sedimentation ponds or to the river. This is to avoid conditions in the flocculent layer switching from a situation favouring denitrification to one favouring anaerobic fermentation. Through denitrification, a large fraction of the waste is volatilized in situ, keeping discharge $\mathrm{kg}^{-1}$ fish produced low (Phu \& Tinh 2012).

Assuming that $2.86 \mathrm{~g}$ of COD is used to remove $1 \mathrm{~g}$ $\mathrm{NO}_{3}-\mathrm{N}$ by denitrification, and that oxidation of $1 \mathrm{~g}$ organic matter $(\mathrm{OM})$ requires $1.42 \mathrm{~g}$ oxygen (Henze et al. 1997), on average 14698 and $14818 \mathrm{~kg}$ of OM (30 to $39 \mathrm{~g}$ COD or 21 to $27 \mathrm{~g} \mathrm{OM} \mathrm{kg}^{-1}$ feed) was removed from the DS and US ponds, respectively (Tables $5 \& 7$ ). When averaging the whole pond area, 1.72 and $1.98 \mathrm{~g} \mathrm{NO}_{3}-\mathrm{N} \mathrm{m}^{-2} \mathrm{~d}^{-1}$ was removed daily in US and DS ponds, respectively. This corresponds to 4.95 and $5.66 \mathrm{~g} \mathrm{COD} \mathrm{removal} \mathrm{m}^{-2} \mathrm{~d}^{-1}$, and 0.11 and 0.13 equivalents of alkalinity production $\mathrm{m}^{-2} \mathrm{~d}^{-1}$ $\left(14 \mathrm{~g} \mathrm{NO}_{3}-\mathrm{N}\right.$ removal $=0.91$ alkalinity equivalents; Henze et al. 1997) in US and DS ponds, respectively. 
The alkalinity production compensated for a daily application of 9.1 and $10.8 \mathrm{~g} \mathrm{NaHCO}_{3} \mathrm{~m}^{-2} \mathrm{~d}^{-1}$ (1 alkalinity equivalent $=83 \mathrm{~g} \mathrm{NaHCO}_{3}$ ) in US and DS ponds, respectively.

\section{Sustainability indicators}

Fingerling use

The mortality (calculated as the difference between the number of fingerlings stocked and fish harvested) of striped catfish in this study was higher than reported for channel catfish production in ponds, trout in raceways and tilapia in recirculation systems (Table 10). As discussed in the previous section, reducing disease-related mortality is important. Because mortality occurs mainly during the first 2 mo of the production cycle and fingerlings are cheap, farmers do not consider lowering mortality a priority. However, when considering the drop in the number of feeding days, the high variation in feed input (Fig. 2) and the longer culture period (see previous section), then the lack of disease-free fingerlings and the higher than necessary feed conversion ratio turns out to be costly to the industry. Developing culture methods to produce fingerlings using alternative methods, such as in closed recirculation systems, could be instrumental in producing disease-free and high quality fingerlings. This needs to coincide with avoiding contamination during transport to the grow-out ponds.

\section{Water use}

Water use per $\mathrm{kg}$ production in aquaculture varies over a broad range, from 0.15 to more than $100 \mathrm{~m}^{3}$ $\mathrm{kg}^{-1}$ fish produced (Verdegem \& Bosma 2009). In this study, water use $\mathrm{kg}^{-1}$ striped catfish production was
20-77 times lower than for trout production in raceways, and 3-14 times smaller than for intensive shrimp ponds (Table 10). Water use in semi-intensive channel catfish ponds was similar to striped catfish production in the Mekong Delta. The water use is further reduced in recirculating aquaculture systems (RAS), where it was 12-30 times lower than for striped catfish pond culture (Table 10) (Verreth \& Oberdieck 2009).

\section{Energy use}

On-farm energy use to produce $1 \mathrm{~kg}$ of striped catfish was 6-113 times lower than the other cultures listed in Table 10. This shows that culturing an airbreathing species in ponds with tidal water exchange requires considerably less on-farm energy input than any other type of fish culture (Boyd \& Tucker 1998, Boyd \& Gross 2000, d'Orbcastel et al. 2009a, Verreth \& Oberdieck 2009).

\section{$\mathrm{N}$ utilization efficiency}

The $\mathrm{N}$ utilization efficiency of the feed was higher for striped catfish than for channel catfish, shrimp, tilapia and trout culture (Table 10). Better N utilization efficiencies were obtained by switching to RAS and using high quality feed (Table 10). N-discharge per $\mathrm{kg}$ fish produced from striped catfish ponds was 1.6-6.8 times lower than for other cultures listed in Table 10. These differences in discharge when compared to other species are much higher than for $\mathrm{N}$ utilization efficiency. A unique feature of the 4-6 m deep striped catfish ponds is the occurrence of denitrification at the bottom, which significantly reduced $\mathrm{N}$ discharge (see 'Water quality maintenance for fish growth' above).

Table 10. Key sustainability indicators of different culture species. RAS: recirculating aquaculture system; FT: flow-through system. Superscript letters indicate source: (a) this study, (b) Gross et al. (1998), (c) Boyd (2005), (d) Boyd et al. (2000), (e) Gross et al. (2000), (f) Verreth \& Oberdieck (2009), (g) d'Orbcastel et al. (2009b), (h) Foy \& Rosell (1991), (i) Warrer-Hansen (1982), Sumari (1982) and Solbe (1982), (j) Thakur \& Lin (2003), (k) Yoo \& Boyd (1994), (l) Boyd \& Tucker (1998), (m) Hall et al. (1992) with commercial feed

\begin{tabular}{|c|c|c|c|c|c|c|c|c|c|}
\hline Species & $\begin{array}{l}\text { Culture } \\
\text { system }\end{array}$ & $\begin{array}{l}\text { Mortality } \\
(\%)\end{array}$ & $\begin{array}{l}\text { Water use } \\
\left(\mathrm{m}^{3} \mathrm{~kg}^{-1} \text { fish }\right)\end{array}$ & $\begin{array}{c}\text { Energy use } \\
\left(\mathrm{kWh} \mathrm{kg}^{-1} \text { fish }\right)\end{array}$ & $\begin{array}{l}\text { Labour use } \\
\left(\mathrm{h} \mathrm{kg}^{-1} \text { fish) }\right.\end{array}$ & $\begin{array}{l}\mathrm{N} \text { retained } \\
\text { (\% of input) }\end{array}$ & $\begin{array}{l}\mathrm{P} \text { retained } \\
\text { (\% of input) }\end{array}$ & $\begin{array}{l}\text { N discharge } \\
\left(\mathrm{g} \mathrm{kg}^{-1} \text { fish) }\right.\end{array}$ & $\begin{array}{l}\text { P discharge } \\
\left(\mathrm{g} \mathrm{kg}^{-1} \text { fish) }\right.\end{array}$ \\
\hline Striped catfish & Pond & $23.6-49^{(\mathrm{a})}$ & $2.8-7.1^{(\mathrm{a})}$ & $0.04-0.14^{(\mathrm{a})}$ & $0.06-0.11^{(\mathrm{a})}$ & $43.7-44.3^{(\mathrm{a})}$ & $17.6-17.7^{(\mathrm{a})}$ & $19.8-20.1^{(a)}$ & $17.0-17.7^{(\mathrm{a})}$ \\
\hline Channel catfish & Pond & $6^{(\mathrm{b})}$ & 3. $7^{(\mathrm{c})}$ & $0.9^{(\mathrm{d})}$ & - & $18.3-34.8^{(\mathrm{e})}$ & $20.4^{(\mathrm{e})}$ & $29.1^{(\mathrm{b})}$ & $10.6-15.4^{(\mathrm{b})}$ \\
\hline Tilapia & RAS & $0.5^{(\mathrm{f})}$ & $0.24^{(\mathrm{f})}$ & $1.8^{(\mathrm{f})}$ & $0.013^{(\mathrm{f})}$ & $32^{(\mathrm{f})}$ & $43^{(f)}$ & $45.9^{(\mathrm{f})}$ & $8.3^{(\mathrm{f})}$ \\
\hline Trout & FT & $0.1^{(\mathrm{g})}$ & $148-215^{(\mathrm{h})}$ & $1-1.7^{(\mathrm{g})}$ & - & $18.9^{(\mathrm{h})}$ & $13.2^{(\mathrm{h})}$ & $73.3-124.2^{(\mathrm{h}, \mathrm{i})}$ & $11.0-25.6^{(\mathrm{h}, \mathrm{i})}$ \\
\hline Intensive shrimp & Pond & $22-50^{(j)}$ & $20-40^{(\mathrm{k})}$ & $4.5^{(1)}$ & - & $22.8-30.7^{(\mathrm{j})}$ & $10.5-12.8^{(\mathrm{j})}$ & $36.5-102^{(j)}$ & $11.6-18^{(j)}$ \\
\hline Salmon & Cages & - & - & - & - & $28.2-30.0^{(\mathrm{m})}$ & $17.7-19.6^{(\mathrm{m})}$ & $80.0-84.7^{(\mathrm{m})}$ & $16.7-18.9^{(\mathrm{m})}$ \\
\hline
\end{tabular}


At present, the industry discharges water effluent directly to the river and sludge to a sedimentation pond or to the river. If all sludge were treated before discharge, then striped catfish farming would actually remove OM from the river. This would not be the case for $\mathrm{N}$ and $\mathrm{P}$, because the majority of these nutrients are discharged with the water effluent and sludge. In a lab scale experiment, Anh \& Mai (2009) reduced the TSS load in the effluent by nearly $20 \%$ by passing it through a stabilization pond operated with a retention time of $1 \mathrm{~h}$.

\section{P utilization efficiency}

The P utilization efficiency of the feed in striped catfish ponds was better than for shrimp or trout. However, the opposite was true for channel catfish and tilapia in RAS. The use of plant ingredients in striped catfish feeds possibly lowered the P available for fish growth (Gatlin et al. 2007, Cao et al. 2008, Kumar et al. 2012, Hung et al. 2015). Nevertheless, $P$ discharge per kg striped catfish produced was similar to the intensive culture of trout, channel catfish and shrimp, and higher than for tilapia in RAS (Table 10). In semi-extensive polyculture ponds, a larger fraction of $\mathrm{P}$ accumulated in the sediment (Nhan et al. 2006, 2008). In the deep striped catfish ponds, $58-60 \%$ of the metabolic waste of $\mathrm{P}$ accumulated in the system or was taken out during sludge removal. If this $\mathrm{P}$ could be trapped in sedimentation ponds (Anh et al. 2010), then transforming this into fertilizer would become an option (Phung et al. 2009, Da et al. 2015). In addition, reducing the $\mathrm{P}$ content in the feed and improving P availability, as was done in trout feeds (Ketola \& Richmond 1994), could also contribute to a further reduction of $\mathrm{P}$ discharge.

\section{CONCLUSIONS AND RECOMMENDATIONS}

When considering the sustainability indicators of energy use, labour use and $\mathrm{N}$ and $\mathrm{P}$ retention and discharge, striped catfish production in deep ponds in the Mekong Delta performed well in comparison to other aquaculture species and systems (Table 10). By developing the 2-6 $\mathrm{m}$ deep pond culture systems, striped catfish farmers made maximum use of the unique topography and water regime in the Mekong Delta, allowing them to keep on-farm energy and labour inputs low, while realizing nutrient efficiencies that compare well to other major aquaculture species. This in part explains the success of striped catfish farming. Nevertheless, further improvements remain possible in both US and DS ponds. Most resource utilization indicators in US ponds were higher than the DS ponds, except for fingerling use. In addition, most nutrient utilization efficiency and nutrient discharge indicators were similar in both DS and US ponds. For improving sustainability, striped catfish culture in earthen ponds can be integrated with algae ponds or helophytic filters to treat influent and effluent water. This way, striped catfish farming uses more land, which is a scarce commodity in the Mekong Delta. Another option is to utilize RAS technology, which would allow farmers to fully control waste streams resulting from culture (d'Orbcastel et al. 2009a). An additional advantage of culturing in RAS would be the reduction in water exchange with the Mekong River, which would minimize horizontal transmission of pollutants, parasites or diseases originating from other farms along the Mekong River and adjacent lands. A major challenge for developing striped catfish culture in RAS is to keep production costs sufficiently low to compete with pond culture in the Mekong Delta.

Acknowledgements. The authors thank 2 striped catfish farms for collaboration, and Mr. Nguyen Van Huynh and Mr. Duong Dinh Nam who collected data during the production cycle. Specially, we are grateful to the Ministry of Agriculture and Rural Development (MARD), Vietnam that supported funding for this study.

\section{LITERATURE CITED}

Adhikari S, Pani KC, Mishra B, Jena JK, Jayasankar P (2014) Carbon, nitrogen and phosphorus budget for the culture of Indian major carps with different stocking density. Hydrol Curr Res 5:176

Almazan G, Boyd CE (1978) An evaluation of Secchi disk visibility for estimating plankton density in fish ponds. Hydrobiologia 61:205-208

Anh PT, Mai HNP (2009) Identification, evaluation and preparation guide book on wastewater treatment methods for pangasius farms. Report No. MOFI/FSPS-II SUDA/2008/3221 Smartchoice educational and environmental consultants. Prepared for Sustainable Development of Aquaculture (SUDA), Ministry of Agriculture and Rural Development (MARD), Hanoi

Anh PT, Kroeze C, Bush SR, Mol APJ (2010) Water pollution by pangasius production in the Mekong Delta, Vietnam: causes and options for control. Aquacult Res 42:108-128

AOAC (Association of Official Analytical Chemists) (2000) Official methods of analysis of the Association of Official Analytical Chemists. AOAC, Washington, DC

APHA (American Public Health Association) (1999) Standard methods for the examination of water and waste water, 20th edn. American Public Health Association, American Water Works Association, Water Pollution Control Federation, Washington, DC 
ASC (Aquaculture Stewardship Council) (2012) ASC pangasius standard. www.asc-aqua.org/upload/ASC\%20Pangasius \%20Standard_v1.0.pdf (assessed 15 Jun 2016)

Bondad-Reantaso MG, Subasinghe RP, Arthur JR, Ogawa K and others (2005) Disease and health management in Asian aquaculture. Vet Parasitol 132:249-272

Bosma RH, Chau TH, José P (2009) Environmental impact assessment of the pangasius sector in the Mekong Delta. Report to the Dutch Ministry of Agriculture, Nature and Food Quality, and the Vietnamese Ministry of Agriculture and Rural Development. Wageningen University, Wageningen

Boyd CE (1985) Chemical budgets for channel catfish ponds. Trans Am Fish Soc 114:291-298

Boyd CE (2005) Water use in aquaculture. World Aquacult $36: 12-15,70$

Boyd CE, Gross A (2000) Water use and conservation for inland aquaculture ponds. Fish Manag Ecol 7:55-63

Boyd CE, Tucker CS (1998) Pond aquaculture water quality management. Kluwer Academic Publishers, Boston, MA

Boyd CE, Queiroz J, Lee J, Rowan M, Whitis GN, Gross A (2000) Environmental assessment of channel catfish Ictalurus punctatus farming in Alabama. J World Aquacult Soc 31:511-544

Cao L, Yang Y, Wang WM, Yakupitiyage A, Yuan DR, Diana JS (2008) Effects of pretreatment with microbial phytase on phosphorous utilization and growth performance of Nile tilapia (Oreochromis niloticus). Aquacult Nutr 14: 99-109

d'Orbcastel ER, Blancheton JP, Aubin J (2009a) Towards environmentally sustainable aquaculture: comparison between two trout farming systems using life cycle assessment. Aquacult Eng 40:113-119

d'Orbcastel ER, Blancheton JP, Belaud A (2009b) Water quality and rainbow trout performance in a Danish model farm recirculating system: comparison with a flow through system. Aquacult Eng 40:135-143

Da CT, Phuoc LH, Duc HN, Troell M, Berg H (2015) Use of wastewater from striped catfish (Pangasianodon hypophthalmus) pond culture for integrated rice-fish-vegetable farming systems in the Mekong Delta, Vietnam. Agroecol Sustain Food Syst 39:580-597

Dalsgaard J, Pedersen PB (2011) Solid and suspended/ dissolved waste $(\mathrm{N}, \mathrm{P}, \mathrm{O})$ from rainbow trout (Oncorynchus mykiss). Aquaculture 313:92-99

De Silva SS, Brett AI, Phuong TN, Tam MB, Geoff JG, Giovanni MT (2010) Estimation of nitrogen and phosphorus in effluent from the striped catfish farming sector in the Mekong Delta, Vietnam. Ambio 39:504-514

* Desortová B (1981) Relationship between chlorophyll a concentration and phytoplankton biomass in several reservoirs in Czechoslovakia. Int Rev Gesamten Hydrobiol Hydrograph 66:153-169

Drapcho CM, Brune DE (2000) The partitioned aquaculture system: impact of design and environmental parameters on algal productivity and photosynthetic oxygen production. Aquacult Eng 21:151-168

Eding EH, Kamstra A (2002) Netherlands farms tune recirculation systems to production of varied species. Global Aquacult Advocate 5:52-54

Eding EH, Weerd VD (1999) Grundlagen, Aufbau und Management von Kreislaufanlagen. In: Bohl M (ed) Zucht und Produktion von Süsswasserfischen. DLG-Verlagsgesellschaft, Frankfurt, p 436-491

Foy RH, Rosell R (1991) Loadings of nitrogen and phospho- rus from a Northern Ireland fish farm. Aquaculture 96: $17-30$

*Funge-Smith SJ, Briggs MRP (1998) Nutrient budgets in intensive shrimp ponds: implications for sustainability. Aquaculture 164:117-133

Gatlin DM, Barrows FT, Brown P, Dabrowski K and others (2007) Expanding the utilization of sustainable plant products in aquafeeds: a review. Aquacult Res 38: $551-579$

Gross A, Boyd CE, Lovell RT, Eya JC (1998) Phosphorus budgets for channel catfish ponds receiving diets with different phosphorus concentrations. J World Aquacult Soc 29:31-39

Gross A, Boyd CE, Wood CW (2000) Nitrogen transformations and balance in channel catfish ponds. Aquacult Eng 24:1-14

*Hall POJ, Holby O, Kollberg S, Samuelsson MO (1992) Chemical fluxes and mass balances in a marine fish cage farm. IV. Nitrogen. Mar Ecol Prog Ser 89:81-91

*Henken A, Lucas H, Tijssen P, Machiels M (1986) A comparison between methods used to determine the energy content of feed, fish and faeces sample. Aquaculture 58: 195-201

Henze M, Harremoes P, Jansen J, Arvin E (1997) Wastewater treatment: biological and chemical process, 2nd edn. Springer, Berlin

*Hung LT, Thanh NT, Pham MA, Browdy CL (2015) A comparison of the effect of dietary fungal phytase and dicalcium phosphate supplementation on growth performances, feed and phosphorus utilization of tra catfish juveniles (Pangasianodon hypophthalmus Sauvage, 1878). Aquacult Nutr 21:10-17

Huong DTT, Quyen MD, Lefevre S, Wang T, Bayley M (2011) Study on the physiological and hematological changes of stripped catfish (Pangasianodon hypophthalmus) fingerling exposed to different nitrite concentrations. In: Phuong NT, Phu TQ, Hien TT, Tuan NA and others (eds) Proc 4th Aquacult Fish Conf, CanTho University, 26 Jan 2011, CanTho, Vietnam. Agricultural Publishing House, Ho Chi Minh City, p 166-177

Ketola HG, Richmond ME (1994) Requirement of rainbow trout for dietary phosphorus and its relationship to the amount discharged in hatchery effluents. Trans Am Fish Soc 123:587-594

Kitson RE, Mellon MG (1944) Colorimetric determination of phosphorus as molybdivanadophosphoric acid. Ind Eng Chem Anal Ed 16:379-383

K Kumar V, Sinha AK, Makkar HPS, De Boeck G, Becker K (2012) Phytate and phytase in fish nutrition. J Anim Physiol Anim Nutr (Berl) 96:335-364

Lefevre S, Huong DTT, Ha NTK, Wang T, Phuong NT, Bayley $M$ (2011a) A telemetry study of swimming depth and oxygen level in a pangasius pond in the Mekong Delta. Aquaculture 315:410-413

Lefevre S, Huong DTT, Wang T, Phuong NT, Bayley M (2011b) Hypoxia tolerance and partitioning of bimodal respiration in the striped catfish (Pangasianodon hypophthalmus). Comp Biochem Physiol A Mol Integr Physiol 158:207-214

Lin C, Yang Y, Diana J (1997) The effects of pond management strategies on nutrient budgets: Thailand. In: Burke D, Goetze B, Clair D, Egna H (eds) Fourteenth annual technical report. Pond Dynamics/Aquaculture Collaborative Research Support Program, Oregon State University, Corvallis, OR, p 19-24 
Linh NTT (2012) Effect of $\mathrm{H}_{2} \mathrm{~S}$ on growth, survival and meat color of striped catfish (Pangasianodon hypophthalmus). Sci Technol J Travinh Univ 4:29-33 (in Vietnamese)

MARD (Ministry of Agriculture and Rural Development) (2003) National technical regulation on commercial striped catfish culture farm in ponds - conditions for veterinary hygiene, environmental protection and food safety. MARD, Ha Noi (in Vietnamese)

MARD (2016) Annual report of striped catfish production in Mekong Delta, Vietnam. MARD, Ha Noi (in Vietnamese)

Mill HR (1907) The best form of rain gauge, with notes on other forms. QJR Meteorol Soc 33:265-274

Muendo PN, Stoorvogel J, Gamal EN, Verdegem M (2005) Rhizons improved estimation of nutrient losses because of seepage in aquaculture ponds. Aquacult Res 36:1333-1336

Nhan DK, Milstein A, Verdegem M, Verreth J (2006) Food inputs, water quality and nutrient accumulation in integrated pond systems: a multivariate approach. Aquaculture 261:160-173

Nhan DK, Verdegem M, Milstein A, Verreth J (2008) Water and nutrient budgets of ponds in integrated agriculture-aquaculture systems in the Mekong Delta, Vietnam. Aquacult Res 39:1216-1228

Phan LT, Bui TM, Nguyen TTT, Gooley GJ and others (2009) Current status of farming practices of striped catfish, Pangasianodon hypophthalmus in the Mekong Delta, Vietnam. Aquaculture 296:227-236

Phu TQ, Tinh TK (2012) Chemical compositions of sludge from intensive striped catfish (Pangasianodon hypophthalmus) culture pond. Sci J CanTho Univ 22:290-299 (in Vietnamese)

Phung CV, Phuc NB, Hoang TK, Bell RW (2009) Recycling of fishpond waste for rice cultivation in the Cuu Long Delta, Vietnam. In: Nair J, Furedy C, Hoysala C, Doelle J (eds) Technologies and management for sustainable biosystems. Nova Science Publishers, New York, NY, p 87-93
Phuong NT, Oanh D (2010) Striped catfish aquaculture in Vietnam: a decade of unprecedented development. In: De Silva SS, Davy FB (eds) Success stories in Asian aquaculture. Springer, Dordrecht, p 131-147

Sang NV, Klemetsdal G, Ødegård J, Gjøen HM (2012) Genetic parameters of economically important traits recorded at a given age in striped catfish (Pangasianodon hypophthalmus). Aquaculture 344-349:82-89

Solbe JFLG (1982) Fish farm effluents: a United Kingdom survey. In: Alabaster JS (ed) Report of the EIFAC workshop on fish farm effluents, Silkeborg, Demark. European Inland Fisheries Advisory Commission (EIFAC) Tech Pap No. 41, FAO, Rome, p 29-55

Sumari O (1982) A report on fish farm effluents in Finland. In: Alabaster JS (ed) Report of the EIFAC workshop on fish farm efflents, Silkeborg, Denmark. EIFAC Tech Pap No. 41, FAO, Rome, p 21-27

Thakur DP, Lin CK (2003) Water quality and nutrient budget in closed shrimp (Penaeus monodon) culture systems. Aquacult Eng 27:159-176

*Verdegem MCJ, Bosma RH (2009) Water withdrawal for brackish and inland aquaculture, and options to produce more fish in ponds with present water use. Water Policy 11:52-68

* Verdegem MCJ, Bosma RH, Verreth JAJ (2006) Reducing water use for animal production through aquaculture. Int J Water Resour Dev 22:101-113

Verreth JAV, Oberdieck A (2009) A handbook for sustainable aquaculture. Project No: COLL-CT-2006-030384. Eurofish, Copenhagen

Warrer-Hansen I (1982) Evaluation of matter discharged from farming in Denmark. In: Alabaster JS (ed) Report of EIFAC workshop on fish farm effluents, Silkeborg, Denmark. EIFAC Tech Pap No. 41, FAO, Rome, p 57-63

Yoo KH, Boyd CE (1994) Hydrology and water supply for aquaculture. Chapman \& Hall, New York, NY 


\section{Appendix}


whole initial fish body $(\%)$, inflow water $\left(\mathrm{g} \mathrm{m}^{-3}\right)$, outflow water $\left(\mathrm{g} \mathrm{m}^{-3}\right)$, feed $(\%)$, seepage water $\left(\mathrm{g} \mathrm{m}^{-3}\right)$, in sludge removal frequency during culture cycle $\left(\mathrm{g} \mathrm{m}^{-3}\right)$, remained DM of sludge in pond at harvest day $(\%)$. $E_{\text {electricity: total electricity consumption }}$ per culture cycle $(\mathrm{kWh})$. FW: weight of complete skinless fillet after removing fat and red muscle following standard process for export market $(\mathrm{g}) ; \mathrm{FC}$ : cumulative feed $(\mathrm{kg}) ; L_{\text {time }}$ : total time use of labour during whole culture cycle (hours). $M_{\mathrm{tot} . c h e m i c a l}$ : total amount of chemical (as lime, $\mathrm{NaCl}, \mathrm{CuSO}_{4}$ or iodine) during whole culture cycle $(\mathrm{g}) ; M_{\text {tot.antibiotic }}$ : total antibiotic use during whole culture cycle $(\mathrm{g}) ; M_{\text {tot.sludge }}$ : total remaining DM of sludge in pond at harvest day (kg). $\mathrm{n}_{\text {white }}$ : number of white fillet fish; $\mathrm{n}_{\text {pink }}$ : number of pink fillet fish (no.); $\mathrm{n}_{\text {yellow }}$ : number of yellow fillet fish (no.); $\mathrm{n}$ : number of fish samples (no.). $V_{\text {tot,inflow: }}$ total amount of inflow water during culture cycle $\left(\mathrm{m}^{3}\right)_{;} V_{\text {oil }}$ : total volume of diesel oil utilization during whole culture cycle (l); $V_{\text {tot.outflow: }}$ total volume of outflow water $\left(\mathrm{m}^{3}\right)_{i} V_{\text {tot.seepage: }}$ total volume of seepage water $\left(\mathrm{m}^{3}\right) ; V_{\text {tot.sludge removal frequency }}$ t total volume of sludge removal frequency during culture cycle $\left(\mathrm{m}^{3}\right) . W_{\text {Final sample: }}$ fish biomass of sample at harvest $(\mathrm{kg}) ; W_{\text {tot.Initial }}$ total initial fish biomass at stocking day by weight $(\mathrm{kg}) ; W_{\text {tot.Final }}$ : total fish biomass at harvest day by weight $(\mathrm{kg}) ; W_{\text {Initial sample: initial fish }}$ biomass of sample $(\mathrm{kg}) . X_{\text {fish retained }}$ DM, N or P retained in fish $(\mathrm{g}) ; X_{\text {discharge: }}$ DM, N or P discharge $(\mathrm{kg})$

\begin{tabular}{|c|c|c|}
\hline Parameter & Unit & Formula \\
\hline \multicolumn{3}{|l|}{ Fish performance } \\
\hline Initial individual body weight ( $\left.W_{\text {Initial }}\right)$ & g & $W_{\text {Initial }}=W_{\text {Initial sample }} / \mathrm{n}$ \\
\hline Final individual body weight $\left(W_{\text {Final }}\right)$ & $g$ & $W_{\text {Final }}=W_{\text {Final sample }} / \mathrm{n}$ \\
\hline Total fish stocked in pond ( $\left.N_{\text {tot.Initial }}\right)$ & No. & $N_{\text {tot.Initial }}=1000 \times W_{\text {tot.Initial }} / W_{\text {Initial }}$ \\
\hline Total fish harvested from pond ( $\left.N_{\text {tot.Final }}\right)$ & No. & $N_{\text {tot.Final }}=1000 \times W_{\text {tot.Final }} / W_{\text {Final }}$ \\
\hline Survival $(S)$ & $\%$ & $S=100 \times\left(N_{\text {tot.Final }} / N_{\text {tot.Initial }}\right)$ \\
\hline Specific growth rate (SGR) & $\% \mathrm{BW} \mathrm{d}^{-1}$ & $\mathrm{SGR}=100 \times\left(\ln W_{\text {Final }}-\ln W_{\text {Initial }}\right) /$ Day \\
\hline Total biomass gain $\left(G_{\mathrm{tot}}\right)$ & $\mathrm{kg}$ & $G_{\text {tot }}=W_{\text {tot.Final }}-W_{\text {tot.Initial }}$ \\
\hline Feed conversion ratio (FCR) & $\mathrm{kg} \mathrm{kg}^{-1}$ & $\mathrm{FCR}=\mathrm{FC} / G_{\mathrm{tot}}$ \\
\hline Biomass of fish mortality $\left(G_{\text {fish.mortality }}\right)$ & $\mathrm{kg}$ & Daily accumulation \\
\hline Fillet yield (FY) & $\%$ & $\mathrm{FY}=100 \times\left(\mathrm{FW} / W_{\text {Final }}\right)$ \\
\hline Fillet colour grade (FIG) & & FIG $=\left(1 \times \mathrm{n}_{\text {white }}+2 \times \mathrm{n}_{\text {pink }}+3 \times \mathrm{n}_{\text {yellow }}\right) / \mathrm{n}$ \\
\hline \multicolumn{3}{|l|}{ Nutrient mass balances } \\
\hline \multicolumn{3}{|l|}{ At fish level } \\
\hline Nutrients in feed ( $\left.X_{\text {feed }}\right)$ & $\mathrm{kg}$ & $X_{\text {feed }}=\left(C_{X \text {.feed }} / 100\right) \times \mathrm{FC}$ \\
\hline Nutrients in fish retained ( $X_{\text {fish retained }}$ ) & $\mathrm{kg}$ & $X_{\text {fish retained }}=\left(C_{X \text {.Final fish }} \times W_{\text {tot.Final }}\right)-\left(C_{X . \text { Initial fish }} \times W_{\text {tot.Initial }}\right)$ \\
\hline Nutrients in fish mortality $\left(X_{\text {mortality }}\right)$ & $\mathrm{kg}$ & $X_{\text {mortality }}=C_{X . \text { fish.mortality }} \times G_{\text {fish.mortality }}$ \\
\hline Metabolic waste production $\left(X_{\mathrm{MW}}\right)$ & $\mathrm{kg}$ & $X_{\mathrm{MW}}=X_{\text {feed }}-X_{\text {fish retained }}-X_{\text {mortality }}$ \\
\hline \multicolumn{3}{|l|}{ At pond level } \\
\hline Nutrient input ( $\left.X_{\text {input pond }}\right)$ & $\mathrm{kg}$ & $X_{\text {input pond }}=X_{\mathrm{MW}}+X_{\text {influent }}$ \\
\hline Nutrient influent water $\left(X_{\text {influent }}\right)$ & $\mathrm{kg}$ & $X_{\text {influent }}=\left(C_{X . \text { inflow }} \times \mathrm{V}_{\text {tot.inflow }}\right) / 1000$ \\
\hline Nutrient output ( $\left.X_{\text {output pond }}\right)$ & $\mathrm{kg}$ & $X_{\text {output pond }}=X_{\text {effluent }}+X_{\text {sludge }}+X_{\text {unaccounted }}$ \\
\hline Nutrient in effluent $\left(X_{\text {effluent }}\right)$ & $\mathrm{kg}$ & $X_{\text {effluent }}=\left[\left(C_{X \text {.outflow }} \times V_{\text {tot.outflow }}\right)+\left(C_{X \text {.seepage }} \times V_{\text {tot.seepage }}\right)\right] / 1000$ \\
\hline Nutrient in sludge $\left(X_{\text {sludge }}\right)$ & $\mathrm{kg}$ & $\begin{array}{l}X_{\text {sludge }}=\left[C_{X . \text { sludge removal frequency }} \times V_{\text {tot.sludge removal frequency }}\right] / 1000+ \\
\left(C_{X . \text { remained sludge }} \times M_{\text {tot.sludge }}\right) / 100\end{array}$ \\
\hline Nutrient unaccounted ( $\left.X_{\text {unaccounted }}\right)$ & $\mathrm{kg}$ & $X_{\text {unaccounted }}=X_{\text {inputs }}-\left(X_{\text {effluent }}+X_{\text {sludge }}\right)$ \\
\hline \multicolumn{3}{|l|}{ Sustainability indicators ${ }^{a}$} \\
\hline \multicolumn{3}{|l|}{ Resource utilization efficiency } \\
\hline Fingerling use (FU) & $\# \mathrm{~kg}$ fish $^{-1}$ & $\mathrm{FU}=\left(N_{\text {tot Initial }}-N_{\text {tot Final }}\right) / G_{\text {tot }}$ \\
\hline Water use (WU) & $\mathrm{m}^{3} \mathrm{~kg}^{-1}$ fish & $\mathrm{WU}=V_{\text {tot.inflow }} / G_{\mathrm{tot}}$ \\
\hline Diesel use (DU) & $\mathrm{ml} \mathrm{kg}^{-1}$ fish & $\mathrm{DU}=V_{\mathrm{oil}} / G_{\mathrm{tot}}$ \\
\hline Energy use (EU) & $\mathrm{kWh} \mathrm{kg}^{-1}$ fish & $\mathrm{EU}=E_{\text {electricity }} / G_{\text {tot }}$ \\
\hline Labour use (LU) & $\mathrm{h} \mathrm{kg}^{-1}$ fish & $\mathrm{LU}=L_{\text {time }} / G_{\text {tot }}$ \\
\hline Chemical use (CU) & $\mathrm{g} \mathrm{kg}^{-1}$ fish & $\mathrm{CU}=M_{\text {tot.chemical }} / G_{\text {tot }}$ \\
\hline Antibiotic use (AU) & $\mathrm{g} \mathrm{kg}^{-1}$ fish & $\mathrm{AU}=M_{\text {tot.antibiotic }} / G_{\text {tot }}$ \\
\hline \multicolumn{3}{|l|}{ Nutrient utilization efficiency } \\
\hline $\mathrm{XR}(X=\mathrm{DM}$ or $\mathrm{P}$ or $\mathrm{N})$ & $\mathrm{g} \mathrm{kg}^{-1}$ fish & $\mathrm{XR}=1000 \times X_{\text {fish retained }} / G_{\text {tot }}$ \\
\hline \multirow[t]{2}{*}{ Nutrient discharge $(\mathrm{XD})$ : } & $\mathrm{g} \mathrm{kg}^{-1}$ fish & $\mathrm{XD}=1000 \times X_{\text {discharge }} / G_{\mathrm{tot}}$ \\
\hline & & $X_{\text {discharge }}=X_{\text {effluent }}+X_{\text {sludge }}-X_{\text {influent }}$ \\
\hline
\end{tabular}

